\title{
HOMOGENIZATION OF VARIATIONAL PROBLEMS IN MANIFOLD VALUED SOBOLEV SPACES
}

\author{
JeAn-François Babadjian $^{1}$ And Vincent Millot $^{2}$
}

\begin{abstract}
Homogenization of integral functionals is studied under the constraint that admissible maps have to take their values into a given smooth manifold. The notion of tangential homogenization is defined by analogy with the tangential quasiconvexity introduced by Dacorogna et al. [Calc. Var. Part. Diff. Eq. 9 (1999) 185-206]. For energies with superlinear or linear growth, a $\Gamma$-convergence result is established in Sobolev spaces, the homogenization problem in the space of functions of bounded variation being the object of [Babadjian and Millot, Calc. Var. Part. Diff. Eq. 36 (2009) 7-47].
\end{abstract}

Mathematics Subject Classification. 74Q05, 49J45, 49Q20.

Received November 14, 2008.

Published online July 31, 2009.

\section{INTRODUCTION}

The homogenization theory aims to find an effective description of materials whose heterogeneities scale is much smaller than the size of the body. The simplest example is periodic homogenization for which the microstructure is assumed to be periodically distributed within the material. In the framework of the Calculus of Variations, periodic homogenization problems rest on the study of equilibrium states, or minimizers, of integral functionals of the form

$$
\int_{\Omega} f\left(\frac{x}{\varepsilon}, \nabla u\right) \mathrm{d} x, \quad u: \Omega \rightarrow \mathbb{R}^{d}
$$

under suitable boundary conditions, where $\Omega \subset \mathbb{R}^{N}$ is a bounded open set and $f: \mathbb{R}^{N} \times \mathbb{R}^{d \times N} \rightarrow[0,+\infty)$ is some oscillating integrand with respect to the first variable. To understand the asymptotic behavior of (almost) minimizers of such energies, it is convenient to perform a $\Gamma$-convergence analysis (see [13] for a detailed description of this subject) which is an adequate theory to study such variational problems. It is usual to assume that the integrand $f$ satisfies uniform $p$-growth and $p$-coercivity conditions (with $1 \leq p<+\infty$ ) so that one should ask the admissible fields to belong to the Sobolev space $W^{1, p}$. For energies with superlinear growth, i.e., $p>1$, this problem has a quite long history, and we refer to [20] in the convex case. Then it has received the most general answer in the independent works of $[7,21]$, showing that such materials asymptotically behave like homogeneous ones. These results have been subsequently generalized into a lot of different manners. Let us mention [9] where the authors add a surface energy term allowing for fractured media. In that case, Sobolev spaces are not adapted to take into account eventual discontinuities of the deformation field across the cracks.

\footnotetext{
Keywords and phrases. Homogenization, $\Gamma$-convergence, manifold valued maps.

1 CMAP, UMR 7641, École polytechnique, 91128 Palaiseau, France. babadjian@cmap.polytechnique.fr

2 Université Paris Diderot - Paris 7, CNRS, UMR 7598, Laboratoire Jacques-Louis Lions, 75005 Paris, France.

millot@math.jussieu.fr
} 
In many applications admissible fields have to satisfy additional constraints. This is for example the case in the study of equilibria for liquid crystals, in ferromagnetism or for magnetostrictive materials where the order parameters take their values into a given manifold. It then becomes necessary to understand the behaviour of integral functionals of the type (1.1) under this additional constraint. For fixed $\varepsilon>0$, the possible lack of lower semicontinuity of the energy may prevent the existence of minimizers (with eventual boundary conditions). It leads to compute its relaxation under the manifold constraint. In the framework of Sobolev spaces, it has been studied in [1,12], and the relaxed energy is obtained by replacing the integrand by its tangential quasiconvexification which is the analogue of the quasiconvex envelope in the non constrained case. We finally mention a slightly different problem originally introduced in [6,10], where the energy is assumed to be finite only for smooth maps. Recent generalizations can be found in [19] where the study is performed within the framework of Cartesian Currents (see [18]). It shows the emergence in the relaxation process of non local effects of topological nature related to the non density of smooth maps (see $[4,5]$ ).

The aim of this paper is to treat the problem of manifold constrained homogenization, i.e., the asymptotic as $\varepsilon \rightarrow 0$ of energies of the form (1.1) defined on manifold valued Sobolev spaces. Let us make the idea more precise. We consider a connected smooth submanifold $\mathcal{M}$ of $\mathbb{R}^{d}$ without boundary. The tangent space of $\mathcal{M}$ at a point $s \in \mathcal{M}$ will be denoted by $T_{s}(\mathcal{M})$. The class of admissible maps we are interested in is defined as

$$
W^{1, p}(\Omega ; \mathcal{M}):=\left\{u \in W^{1, p}\left(\Omega ; \mathbb{R}^{d}\right): u(x) \in \mathcal{M} \text { for } \mathcal{L}^{N} \text {-a.e. } x \in \Omega\right\} .
$$

For a smooth $\mathcal{M}$-valued map, it is well known that first order derivatives belong to the tangent space of $\mathcal{M}$. For $u \in W^{1, p}(\Omega ; \mathcal{M})$, this property still holds in the sense that $\nabla u(x) \in\left[T_{u(x)}(\mathcal{M})\right]^{N}$ for $\mathcal{L}^{N}$-a.e. $x \in \Omega$.

The energy density $f: \mathbb{R}^{N} \times \mathbb{R}^{d \times N} \rightarrow[0,+\infty)$ is assumed to be a Carathéodory integrand satisfying:

$\left(H_{1}\right)$ for every $\xi \in \mathbb{R}^{d \times N}$ the function $f(\cdot, \xi)$ is 1-periodic, i.e., if $\left\{e_{1}, \ldots, e_{N}\right\}$ denotes the canonical basis of $\mathbb{R}^{N}$, one has $f\left(y+e_{i}, \xi\right)=f(y, \xi)$ for every $i=1, \ldots, N$ and $y \in \mathbb{R}^{N}$;

$\left(H_{2}\right)$ there exist $0<\alpha \leq \beta<+\infty$ and $1 \leq p<+\infty$ such that

$$
\alpha|\xi|^{p} \leq f(y, \xi) \leq \beta\left(1+|\xi|^{p}\right) \quad \text { for a.e. } y \in \mathbb{R}^{N} \text { and all } \xi \in \mathbb{R}^{d \times N} .
$$

For $\varepsilon>0$, we define the functionals $\mathcal{F}_{\varepsilon}: L^{p}\left(\Omega ; \mathbb{R}^{d}\right) \rightarrow[0,+\infty]$ by

$$
\mathcal{F}_{\varepsilon}(u):= \begin{cases}\int_{\Omega} f\left(\frac{x}{\varepsilon}, \nabla u\right) \mathrm{d} x & \text { if } u \in W^{1, p}(\Omega ; \mathcal{M}), \\ +\infty & \text { otherwise. }\end{cases}
$$

For energies with superlinear growth, we have the following result.

Theorem 1.1. Let $\mathcal{M}$ be a connected smooth submanifold of $\mathbb{R}^{d}$ without boundary, and $f: \mathbb{R}^{N} \times \mathbb{R}^{d \times N} \rightarrow$ $[0,+\infty)$ be a Carathéodory function satisfying $\left(H_{1}\right)$ and $\left(H_{2}\right)$ with $1<p<+\infty$. Then the family $\left\{\mathcal{F}_{\varepsilon}\right\}_{\varepsilon>0}$ $\Gamma$-converges for the strong $L^{p}$-topology to the functional $\mathcal{F}_{\text {hom }}: L^{p}\left(\Omega ; \mathbb{R}^{d}\right) \rightarrow[0,+\infty]$ defined by

$$
\mathcal{F}_{\text {hom }}(u):= \begin{cases}\int_{\Omega} T f_{\text {hom }}(u, \nabla u) \mathrm{d} x & \text { if } u \in W^{1, p}(\Omega ; \mathcal{M}), \\ +\infty & \text { otherwise }\end{cases}
$$

where for every $s \in \mathcal{M}$ and $\xi \in\left[T_{s}(\mathcal{M})\right]^{N}$,

$$
T f_{\text {hom }}(s, \xi)=\lim _{t \rightarrow+\infty} \inf _{\varphi}\left\{f_{(0, t)^{N}} f(y, \xi+\nabla \varphi(y)) \mathrm{d} y: \varphi \in W_{0}^{1, \infty}\left((0, t)^{N} ; T_{s}(\mathcal{M})\right)\right\}
$$

is the tangentially homogenized energy density.

If the integrand $f$ has a linear growth in the $\xi$-variable, i.e., if $f$ satisfies $\left(H_{2}\right)$ with $p=1$, we assume in addition that $\mathcal{M}$ is compact, and that 
$\left(H_{3}\right)$ there exists $L>0$ such that

$$
\left|f(y, \xi)-f\left(y, \xi^{\prime}\right)\right| \leq L\left|\xi-\xi^{\prime}\right| \quad \text { for a.e. } y \in \mathbb{R}^{N} \text { and all } \xi, \xi^{\prime} \in \mathbb{R}^{d \times N} .
$$

Then the following representation result on $W^{1,1}(\Omega ; \mathcal{M})$ holds:

Theorem 1.2. Let $\mathcal{M}$ be a connected and compact smooth submanifold of $\mathbb{R}^{d}$ without boundary, and $f$ : $\mathbb{R}^{N} \times \mathbb{R}^{d \times N} \rightarrow[0,+\infty)$ be a Carathéodory function satisfying $\left(H_{1}\right)$ to $\left(H_{3}\right)$ with $p=1$. Then the family $\left\{\mathcal{F}_{\varepsilon}\right\}_{\varepsilon>0} \Gamma$-converges for the strong $L^{1}$-topology at every $u \in W^{1,1}(\Omega ; \mathcal{M})$ to $\mathcal{F}_{\text {hom }}: W^{1,1}(\Omega ; \mathcal{M}) \rightarrow[0,+\infty)$, where

and $T f_{\text {hom }}$ is given by (1.2).

$$
\mathcal{F}_{\text {hom }}(u):=\int_{\Omega} T f_{\text {hom }}(u, \nabla u) \mathrm{d} x,
$$

We would like to emphasize that the use of hypothesis $\left(H_{3}\right)$ is not too restrictive. Indeed, the $\Gamma$-limit remains unchanged upon first relaxing the functional $\mathcal{F}_{\varepsilon}$ (at fixed $\varepsilon>0$ ) in $W^{1,1}\left(\Omega ; \mathbb{R}^{d}\right.$ ). It would lead to replace the integrand $f$ by its tangential quasiconvexification which, by virtue of the growth condition $\left(H_{1}\right)$, does satisfy such a Lipschitz continuity assumption (see [12]).

We finally underline that Theorem 1.2 is not completely satisfactory in its present form. Indeed, in the case of an integrand with linear growth, the domain of the $\Gamma$-limit is obviously larger than the Sobolev space $W^{1,1}(\Omega ; \mathcal{M})$ and the analysis has to be performed in the space of functions of bounded variation. In fact Theorem 1.2 is a first step in this direction and the complete study in $B V$-spaces can be found in [3].

The paper is organized as follows. The study of the energy density $T f_{\text {hom }}$ and its main properties are presented in Section 2. A locality property of the $\Gamma$-limit is established in Section 3. The upper bound inequalities in Theorems 1.1 and 1.2 are the object of Section 4. The lower bounds are obtained in Section 5 where the proofs of both theorems are completed.

\section{Notations}

We start by introducing some notations. Let $\Omega$ be a generic bounded open subset of $\mathbb{R}^{N}$. We denote by $\mathcal{A}(\Omega)$ the family of all open subsets of $\Omega$. We write $B^{k}(s, r)$ for the closed ball in $\mathbb{R}^{k}$ of center $s \in \mathbb{R}^{k}$ and radius $r>0, Q:=(-1 / 2,1 / 2)^{N}$ the open unit cube in $\mathbb{R}^{N}$, and $Q\left(x_{0}, \rho\right):=x_{0}+\rho Q$.

The space of real valued Radon measures in $\Omega$ with finite total variation is denoted by $\mathcal{M}(\Omega)$. We denote by $\mathcal{L}^{N}$ the Lebesgue measure in $\mathbb{R}^{N}$. If $\mu \in \mathcal{M}(\Omega)$ and $\lambda \in \mathcal{M}(\Omega)$ is a nonnegative Radon measure, we denote by $\frac{\mathrm{d} \mu}{\mathrm{d} \lambda}$ the Radon-Nikodým derivative of $\mu$ with respect to $\lambda$. By a generalization of Besicovitch Differentiation Theorem (see [2], Prop. 2.2), there exists a Borel set $E$ such that $\lambda(E)=0$ and

$$
\frac{\mathrm{d} \mu}{\mathrm{d} \lambda}(x)=\lim _{\rho \rightarrow 0^{+}} \frac{\mu(Q(x, \rho))}{\lambda(Q(x, \rho))}
$$

for all $x \in \operatorname{Supp} \mu \backslash E$.

\section{Properties of the homogenized energy Density}

In this section we present the main properties of the energy density $T f_{\text {hom }}$ defined in (1.2). We consider the bulk energy density

$$
T f_{\text {hom }}(s, \xi):=\liminf _{t \rightarrow+\infty} \inf _{\varphi}\left\{f_{(0, t)^{N}} f(y, \xi+\nabla \varphi(y)) \mathrm{d} y: \varphi \in W_{0}^{1, \infty}\left((0, t)^{N} ; T_{s}(\mathcal{M})\right)\right\}
$$

defined for $s \in \mathcal{M}$ and $\xi \in\left[T_{s}(\mathcal{M})\right]^{N}$. Our first concern is to show that the lim inf above is actually a limit. To this purpose we shall introduce a new energy density $\bar{f}$ for which we can apply classical homogenization theories. 
For $s \in \mathcal{M}$ we denote by $P_{s}: \mathbb{R}^{d} \rightarrow T_{s}(\mathcal{M})$ the orthogonal projection from $\mathbb{R}^{d}$ into $T_{s}(\mathcal{M})$, and we set

$$
\mathbf{P}_{s}(\xi):=\left(P_{s}\left(\xi_{1}\right), \ldots, P_{s}\left(\xi_{N}\right)\right) \text { for } \xi=\left(\xi_{1}, \ldots, \xi_{N}\right) \in \mathbb{R}^{d \times N} .
$$

Given the Carathéodory integrand $f: \mathbb{R}^{N} \times \mathbb{R}^{d \times N} \rightarrow[0,+\infty)$ satisfying assumptions $\left(H_{1}\right)$ and $\left(H_{2}\right)$ with $1 \leq p<+\infty$, we define $\bar{f}: \mathbb{R}^{N} \times \mathcal{M} \times \mathbb{R}^{d \times N} \rightarrow[0,+\infty)$ by

$$
\bar{f}(y, s, \xi):=f\left(y, \mathbf{P}_{s}(\xi)\right)+\left|\xi-\mathbf{P}_{s}(\xi)\right|^{p} .
$$

The new integrand $\bar{f}$ is a Carathéodory function, and $\bar{f}(\cdot, s, \xi)$ is 1-periodic for every $(s, \xi) \in \mathcal{M} \times \mathbb{R}^{d \times N}$. By assumption $\left(H_{2}\right), \bar{f}$ also satisfies uniform $p$-growth and $p$-coercivity conditions, i.e.,

$$
\alpha^{\prime}|\xi|^{p} \leq \bar{f}(y, s, \xi) \leq \beta^{\prime}\left(1+|\xi|^{p}\right) \quad \text { for every }(s, \xi) \in \mathcal{M} \times \mathbb{R}^{d \times N} \text { and a.e. } y \in \mathbb{R}^{N},
$$

for some constants $0<\alpha^{\prime} \leq \beta^{\prime}<+\infty$.

Proposition 2.1. Let $f: \mathbb{R}^{N} \times \mathbb{R}^{d \times N} \rightarrow[0,+\infty)$ be a Carathéodory integrand satisfying $\left(H_{1}\right)$ and $\left(H_{2}\right)$ with $1 \leq p<+\infty$. Then the following properties hold:

(i) for every $s \in \mathcal{M}$ and $\xi \in\left[T_{s}(\mathcal{M})\right]^{N}$,

$$
T f_{\text {hom }}(s, \xi)=\lim _{t \rightarrow+\infty} \inf _{\varphi}\left\{f_{(0, t)^{N}} f(y, \xi+\nabla \varphi(y)) \mathrm{d} y: \varphi \in W_{0}^{1, \infty}\left((0, t)^{N} ; T_{s}(\mathcal{M})\right)\right\},
$$

and

where

$$
T f_{\text {hom }}(s, \xi)=\bar{f}_{\text {hom }}(s, \xi),
$$

$$
\bar{f}_{\text {hom }}(s, \xi):=\lim _{t \rightarrow+\infty} \inf _{\varphi}\left\{f_{(0, t)^{N}} \bar{f}(y, s, \xi+\nabla \varphi(y)) \mathrm{d} y: \varphi \in W_{0}^{1, \infty}\left((0, t)^{N} ; \mathbb{R}^{d}\right)\right\}
$$

is the usual homogenized energy density of $\bar{f}$ (see, e.g., [8], Chap. 14);

(ii) the function $T f_{\text {hom }}$ is tangentially quasiconvex, i.e., for all $s \in \mathcal{M}$ and all $\xi \in\left[T_{s}(\mathcal{M})\right]^{N}$,

$$
T f_{\mathrm{hom}}(s, \xi) \leq \int_{Q} T f_{\mathrm{hom}}(s, \xi+\nabla \varphi(y)) \mathrm{d} y
$$

for every $\varphi \in W_{0}^{1, \infty}\left(Q ; T_{s}(\mathcal{M})\right)$. In particular $T f_{\mathrm{hom}}(s, \cdot)$ is rank one convex;

(iii) there exists $C>0$ such that

$$
\alpha|\xi|^{p} \leq T f_{\text {hom }}(s, \xi) \leq \beta\left(1+|\xi|^{p}\right),
$$

and

$$
\left|T f_{\text {hom }}(s, \xi)-T f_{\text {hom }}\left(s, \xi^{\prime}\right)\right| \leq C\left(1+|\xi|^{p-1}+\left|\xi^{\prime}\right|^{p-1}\right)\left|\xi-\xi^{\prime}\right|
$$

for every $s \in \mathcal{M}$ and $\xi, \xi^{\prime} \in\left[T_{s}(\mathcal{M})\right]^{N}$.

Proof. Fix $s \in \mathcal{M}$ and $\xi \in\left[T_{s}(\mathcal{M})\right]^{N}$. For any $t>0$, we introduce

$$
T f_{t}(s, \xi):=\inf _{\varphi}\left\{f_{(0, t)^{N}} f(y, \xi+\nabla \varphi) \mathrm{d} y: \varphi \in W_{0}^{1, \infty}\left((0, t)^{N} ; T_{s}(\mathcal{M})\right)\right\}
$$


and

$$
\bar{f}_{t}(s, \xi):=\inf _{\varphi}\left\{f_{(0, t)^{N}} \bar{f}(y, s, \xi+\nabla \varphi) \mathrm{d} y: \varphi \in W_{0}^{1, \infty}\left((0, t)^{N} ; \mathbb{R}^{d}\right)\right\}
$$

By classical results (see, e.g., [8], Prop. 14.4), there exists

$$
\lim _{t \rightarrow+\infty} \bar{f}_{t}(s, \xi) \quad \text { for every } s \in \mathcal{M} \text { and } \xi \in\left[T_{s}(\mathcal{M})\right]^{N} .
$$

Hence to prove (i), it suffices to show that $T f_{t}(s, \xi)=\bar{f}_{t}(s, \xi)$ for every $t>0$. For any $\varphi \in W_{0}^{1, \infty}\left((0, t)^{N} ; T_{s}(\mathcal{M})\right)$, we have

$$
\bar{f}_{t}(s, \xi) \leq f_{(0, t)^{N}} \bar{f}(y, s, \xi+\nabla \varphi) \mathrm{d} y=f_{(0, t)^{N}} f(y, \xi+\nabla \varphi) \mathrm{d} y
$$

since $\xi+\nabla \varphi(y) \in\left[T_{s}(\mathcal{M})\right]^{N}$ for a.e. $y \in(0, t)^{N}$. Taking the infimum over all such $\varphi^{\prime}$ s in the right hand side of the previous inequality yields $\bar{f}_{t}(s, \xi) \leq T f_{t}(s, \xi)$. To prove the converse inequality we pick up $\psi \in W_{0}^{1, \infty}\left((0, t)^{N} ; \mathbb{R}^{d}\right)$ and we set $\tilde{\psi}=P_{s}(\psi)$. One easily checks that $\tilde{\psi} \in W_{0}^{1, \infty}\left((0, t)^{N} ; T_{s}(\mathcal{M})\right)$ and $\nabla \tilde{\psi}=\mathbf{P}_{s}(\nabla \psi)$ a.e. in $(0, t)^{N}$. Therefore

$$
T f_{t}(s, \xi) \leq f_{(0, t)^{N}} f(y, \xi+\nabla \tilde{\psi}) \mathrm{d} y=f_{(0, t)^{N}} f\left(y, \mathbf{P}_{s}(\xi+\nabla \psi)\right) \mathrm{d} y \leq f_{(0, t)^{N}} \bar{f}(y, s, \xi+\nabla \psi) \mathrm{d} y
$$

Then the converse inequality arises taking the infimum over all admissible $\psi$ 's.

By standard results $\bar{f}_{\text {hom }}(s, \cdot)$ is a quasiconvex function for every $s \in \mathcal{M}$ (see, e.g., [8], Thm. 14.5). As a consequence, for any $s \in \mathcal{M}, \xi \in\left[T_{s}(\mathcal{M})\right]^{N}$ and $\varphi \in W_{0}^{1, \infty}\left(Q ; T_{s}(\mathcal{M})\right)$, we have

$$
T f_{\text {hom }}(s, \xi)=\bar{f}_{\text {hom }}(s, \xi) \leq \int_{Q} \bar{f}_{\text {hom }}(s, \xi+\nabla \varphi) \mathrm{d} y=\int_{Q} T f_{\text {hom }}(s, \xi+\nabla \varphi) \mathrm{d} y,
$$

which proves that $T f_{\text {hom }}$ is tangentially quasiconvex. As a consequence of $(2.3)$ and the fact that $\bar{f}_{\text {hom }}(s, \cdot)$ is rank one convex, it follows that $T f_{\text {hom }}(s, \cdot)$ is rank one convex as well.

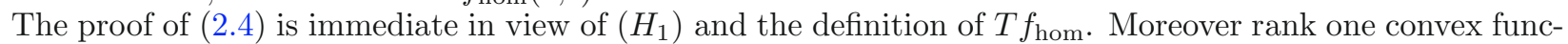
tions satisfying uniform $p$-growth and $p$-coercivity conditions are $p$-Lipschitz (see, e.g., [11], Lem. 2.2, Chap. 4), and thus (2.5) holds.

Remark 2.1. It readily follows from the previous proof that Proposition 2.1 still holds for any Carathéodory integrand $\hat{f}: \mathbb{R}^{N} \times \mathcal{M} \times \mathbb{R}^{d \times N} \rightarrow[0,+\infty)$ instead of $\bar{f}$, provided that: $\hat{f}(x, s, \xi)=f(y, \xi)$ for every $s \in \mathcal{M}$, every $\xi \in\left[T_{s}(\mathcal{M})\right]^{N}$ and a.e. $y \in \mathbb{R}^{N} ; \hat{f}(\cdot, s, \cdot)$ satisfies $\left(H_{1}\right)$ and $\left(H_{2}\right)$ for every $s \in \mathcal{M}$ with uniform estimates with respect to $s$.

Remark 2.2. If $\operatorname{dim}(\mathcal{M})=1$ then $T_{s}(\mathcal{M})$ is a one dimensional linear subspace of $\mathbb{R}^{d}$ for every $s \in \mathcal{M}$. Hence, given $s \in \mathcal{M}$, we can identify $T_{s}(\mathcal{M})$ with $\mathbb{R}$ through some linear mapping $i_{s}: \mathbb{R} \rightarrow T_{s}(\mathcal{M})$. Using the application $i_{s}$, we can also identify $\left[T_{s}(\mathcal{M})\right]^{N}$ with $\mathbb{R}^{N}$ setting for $z=\left(z_{1}, \ldots, z_{N}\right) \in \mathbb{R}^{N}, i_{s}(z):=\left(i_{s}\left(z_{1}\right), \ldots, i_{s}\left(z_{N}\right)\right)$. Define $\hat{f}(y, s, z):=f\left(y, i_{s}(z)\right)$ for $(y, s, z) \in \Omega \times \mathcal{M} \times \mathbb{R}^{N}$. By (2.3) and [8], Remark 14.6, we can replace in formula (1.2) homogeneous boundary conditions by periodic boundary conditions, and the limit as $t \rightarrow+\infty$ by the infimum over all $t \in \mathbb{N}$. Moreover, in the scalar case the homogenization formula can be reduced to a single 
cell formula (see, e.g., [8], Chap. 14). Therefore

$$
\begin{aligned}
T f_{\text {hom }}(s, \xi) & =\inf _{t \in \mathbb{N}} \inf \left\{f_{(0, t)^{N}} f(y, \xi+\nabla \varphi) \mathrm{d} y: \varphi \in W_{\#}^{1, \infty}\left((0, t)^{N} ; T_{s}(\mathcal{M})\right)\right\} \\
& =\inf _{t \in \mathbb{N}} \inf \left\{f_{(0, t)^{N}} \hat{f}\left(y, s, i_{s}^{-1}(\xi)+\nabla \phi\right) \mathrm{d} y: \phi \in W_{\#}^{1, \infty}\left((0, t)^{N}\right)\right\} \\
& =\inf \left\{\int_{Q} \hat{f}\left(y, s, i_{s}^{-1}(\xi)+\nabla \phi\right) \mathrm{d} y: \phi \in W_{\#}^{1, \infty}(Q)\right\} \\
& =\inf \left\{\int_{Q} f(y, \xi+\nabla \varphi) \mathrm{d} y: \varphi \in W_{\#}^{1, \infty}\left(Q ; T_{s}(\mathcal{M})\right)\right\} .
\end{aligned}
$$

This remark states that whenever the manifold $\mathcal{M}$ is one dimensional, test functions in the minimization problem (1.2) are in fact scalar valued, and thus, one can compute the tangentially homogenized energy density over one single cell instead of an infinite set of cells. Note that this is not true in general even in the non constrained case (see, e.g., the counter-example in [21], Thm. 4.3).

We conclude this section with an elementary example where the dependence on the $s$-variable is explicit. It shows that tangential homogenization does not reduce in general to standard homogenization. The construction is based on a rank one laminate for which direct computations can be performed.

Example 2.1. Assume that $\mathcal{M}=\mathbb{S}^{1}$ and for $x \in \mathbb{R}^{N}, \xi=\left(\xi_{i j}\right) \in \mathbb{R}^{2 \times N}$,

$$
f(x, \xi)=\sum_{j=1}^{N}\left(a\left(x_{1}\right)\left|\xi_{1 j}\right|^{2}+b\left(x_{1}\right)\left|\xi_{2 j}\right|^{2}\right),
$$

where $a, b \in L^{\infty}(\mathbb{R})$ are 1-periodic and bounded from below by a positive constant. Arguing as in Remark 2.2 and [13], Example 25.6, one may compute for $s=\left(s_{1}, s_{2}\right) \in \mathbb{S}^{1}$ and $\xi \in\left[T_{s}\left(\mathbb{S}^{1}\right)\right]^{N}$,

$$
T f_{\mathrm{hom}}(s, \xi)=\sum_{j=1}^{N} \alpha_{j}(s)\left(\left|\xi_{1 j}\right|^{2}+\left|\xi_{2 j}\right|^{2}\right),
$$

with

$$
\alpha_{j}(s)= \begin{cases}\left(\int_{-1 / 2}^{1 / 2} \frac{\mathrm{d} t}{a(t) s_{2}^{2}+b(t) s_{1}^{2}}\right)^{-1} & \text { if } j=1, \\ \int_{-1 / 2}^{1 / 2}\left(a(t) s_{2}^{2}+b(t) s_{1}^{2}\right) \mathrm{d} t & \text { otherwise. }\end{cases}
$$

Compare this result with [13], Example 25.6.

To treat the homogenization problem with $p=1$, we will need to extend the function $\bar{f}$ to the whole space $\mathbb{R}^{N} \times \mathbb{R}^{d} \times \mathbb{R}^{d \times N}$. We state in the following lemma our extension procedure.

Lemma 2.1. Assume that $\mathcal{M}$ is compact. Let $f: \mathbb{R}^{N} \times \mathbb{R}^{d \times N} \rightarrow[0,+\infty)$ be a Carathéodory function satisfying $\left(H_{1}\right)$ to $\left(H_{3}\right)$ with $p=1$. Then there exists a Carathéodory function $g: \mathbb{R}^{N} \times \mathbb{R}^{d} \times \mathbb{R}^{d \times N} \rightarrow[0,+\infty)$ such that

and satisfying:

$$
g(y, s, \xi)=f(y, \xi) \quad \text { for } s \in \mathcal{M} \text { and } \xi \in\left[T_{s}(\mathcal{M})\right]^{N},
$$

(i) $g$ is 1-periodic in the first variable; 
(ii) there exist $0<\alpha^{\prime} \leq \beta^{\prime}$ such that

$$
\alpha^{\prime}|\xi| \leq g(y, s, \xi) \leq \beta^{\prime}(1+|\xi|)
$$

for every $(s, \xi) \in \mathbb{R}^{d} \times \mathbb{R}^{d \times N}$ and a.e. $y \in \mathbb{R}^{N}$;

(iii) there exist $C>0$ and $C^{\prime}>0$ such that

$$
\left|g(y, s, \xi)-g\left(y, s^{\prime}, \xi\right)\right| \leq C\left|s-s^{\prime}\right||\xi|,
$$

and

$$
\left|g(y, s, \xi)-g\left(y, s, \xi^{\prime}\right)\right| \leq C^{\prime}\left|\xi-\xi^{\prime}\right|
$$

for every $s, s^{\prime} \in \mathbb{R}^{d}$, every $\xi \in \mathbb{R}^{d \times N}$ and a.e. $y \in \mathbb{R}^{N}$.

Proof. For $\delta_{0}>0$ fixed, let $\mathcal{U}:=\left\{s \in \mathbb{R}^{d}: \operatorname{dist}(s, \mathcal{M})<\delta_{0}\right\}$ be the $\delta_{0}$-neighborhood of $\mathcal{M}$. Choosing $\delta_{0}>0$ small enough, we may assume that the nearest point projection $\Pi: \mathcal{U} \rightarrow \mathcal{M}$ is a well defined Lipschitz mapping. Then the map $s \in \mathcal{U} \mapsto P_{\Pi(s)}$ is Lipschitz. Now we introduce a cut-off function $\chi \in \mathcal{C}_{c}^{\infty}\left(\mathbb{R}^{d} ;[0,1]\right)$ such that $\chi(t)=1$ if $\operatorname{dist}(s, \mathcal{M}) \leq \delta_{0} / 2$, and $\chi(s)=0$ if $\operatorname{dist}(s, \mathcal{M}) \geq 3 \delta_{0} / 4$. We define

$$
\mathbb{P}_{s}(\xi):=\chi(s) \mathbf{P}_{\Pi(s)}(\xi) \text { for }(s, \xi) \in \mathbb{R}^{d} \times \mathbb{R}^{d \times N} .
$$

We consider the integrand $g: \mathbb{R}^{N} \times \mathbb{R}^{d} \times \mathbb{R}^{d \times N} \rightarrow[0,+\infty)$ given by

$$
g(y, s, \xi)=f\left(y, \mathbb{P}_{s}(\xi)\right)+\left|\xi-\mathbb{P}_{s}(\xi)\right| .
$$

One may check that $g$ is a Carathéodory function, that $g(\cdot, s, \xi)$ is 1-periodic for every $(s, \xi) \in \mathbb{R}^{d} \times \mathbb{R}^{d \times N}$, and that $\left(H_{2}\right)$ yields (2.7). Then (2.8) and (2.9) follow from $\left(H_{3}\right)$ and the Lipschitz continuity of $s \mapsto \mathbb{P}_{s}$.

Remark 2.3. In view of (2.6), one may argue exactly as in the proof of (2.3) to show that

$$
T f_{\text {hom }}(s, \xi)=g_{\text {hom }}(s, \xi) \quad \text { for every } s \in \mathcal{M} \text { and } \xi \in\left[T_{s}(\mathcal{M})\right]^{N},
$$

where

$$
g_{\mathrm{hom}}(s, \xi):=\lim _{t \rightarrow+\infty} \inf _{\varphi}\left\{f_{(0, t)^{N}} g(y, s, \xi+\nabla \varphi(y)) \mathrm{d} y: \varphi \in W_{0}^{1, \infty}\left((0, t)^{N} ; \mathbb{R}^{d}\right)\right\} .
$$

Hence upon extending $T f_{\text {hom }}$ by $g_{\text {hom }}$ outside the set $\left\{(s, \xi) \in \mathbb{R}^{d} \times \mathbb{R}^{d \times N}: s \in \mathcal{M}, \xi \in\left[T_{s}(\mathcal{M})\right]^{N}\right\}$, we can tacitly assume $T f_{\text {hom }}$ to be defined over the whole $\mathbb{R}^{d} \times \mathbb{R}^{d \times N}$.

\section{LOCALiZATiON}

In this section we show that a suitable functional larger than the $\Gamma$-limit is a measure. It will allow us to obtain the upper bound on the $\Gamma$-limit (see Lem. 4.1) through the blow-up method introduced in $[15,16]$.

Let us consider an arbitrary sequence $\left\{\varepsilon_{n}\right\} \searrow 0^{+}$. Along this sequence we define the $\Gamma\left(L^{p}\right)$-lower limit $\mathcal{F}: L^{p}\left(\Omega ; \mathbb{R}^{d}\right) \rightarrow[0,+\infty]$ by

$$
\mathcal{F}(u):=\inf _{\left\{u_{n}\right\}}\left\{\liminf _{n \rightarrow+\infty} \mathcal{F}_{\varepsilon_{n}}\left(u_{n}\right): u_{n} \in W^{1, p}(\Omega ; \mathcal{M}), u_{n} \rightarrow u \text { in } L^{p}\left(\Omega ; \mathbb{R}^{d}\right)\right\} .
$$

The idea is to localize the functionals $\left\{\mathcal{F}_{\varepsilon_{n}}\right\}_{n \in \mathbb{N}}$ on the family $\mathcal{A}(\Omega)$ of all open subsets of $\Omega$. For every $u \in L^{p}\left(\Omega ; \mathbb{R}^{d}\right)$ and every $A \in \mathcal{A}(\Omega)$, define

$$
\mathcal{F}_{\varepsilon_{n}}(u, A):= \begin{cases}\int_{A} f\left(\frac{x}{\varepsilon_{n}}, \nabla u\right) \mathrm{d} x & \text { if } u \in W^{1, p}(\Omega ; \mathcal{M}), \\ +\infty & \text { otherwise. }\end{cases}
$$


Given a compact set $\mathcal{K} \subset \mathcal{M}$ and a subsequence $\left\{\varepsilon_{k}\right\}:=\left\{\varepsilon_{n_{k}}\right\} \searrow 0^{+}$, we introduce for $u \in W^{1, p}(\Omega ; \mathcal{M})$ and $A \in \mathcal{A}(\Omega)$,

$$
\begin{aligned}
\mathcal{F}_{\mathcal{K}}^{\left\{\varepsilon_{k}\right\}}(u, A):=\inf _{\left\{u_{k}\right\}}\left\{\limsup _{k \rightarrow+\infty}\right. & \mathcal{F}_{\varepsilon_{k}}\left(u_{k}, A\right): u_{k} \rightarrow u \text { weakly in } W^{1, p}\left(\Omega ; \mathbb{R}^{d}\right), \\
& \left.u_{k} \rightarrow u \text { uniformly and } u_{k}(x)=u(x) \text { whenever } \operatorname{dist}(u(x), \mathcal{K})>1 \text { for a.e. } x \in \Omega\right\} .
\end{aligned}
$$

A key point in the upcoming analysis is the following locality result.

Lemma 3.1. For every $u \in W^{1, p}(\Omega ; \mathcal{M})$, there exists a subsequence $\left\{\varepsilon_{k}\right\}$ such that the set function $\mathcal{F}_{\mathcal{K}}^{\left\{\varepsilon_{k}\right\}}(u, \cdot)$ is the restriction to $\mathcal{A}(\Omega)$ of a Radon measure absolutely continuous with respect to the Lebesgue measure $\mathcal{L}^{N}$.

Proof. From the $p$-growth condition $\left(H_{2}\right)$ we infer that for any subsequence $\left\{\varepsilon_{k}\right\}$,

$$
\mathcal{F}_{\mathcal{K}}^{\left\{\varepsilon_{k}\right\}}(u, A) \leq \beta \int_{A}\left(1+|\nabla u|^{p}\right) \mathrm{d} x
$$

so it remains to prove the existence of a suitable subsequence $\left\{\varepsilon_{k}\right\}$ for which $\mathcal{F}_{\mathcal{K}}^{\left\{\varepsilon_{k}\right\}}(u, \cdot)$ is (the trace of) a Radon measure.

Step 1. We start by proving that for any subsequence $\left\{\varepsilon_{k}\right\}$ the following subadditivity property holds:

$$
\mathcal{F}_{\mathcal{K}}^{\left\{\varepsilon_{k}\right\}}(u, A) \leq \mathcal{F}_{\mathcal{K}}^{\left\{\varepsilon_{k}\right\}}(u, B)+\mathcal{F}_{\mathcal{K}}^{\left\{\varepsilon_{k}\right\}}(u, A \backslash \bar{C})
$$

for every $A, B$ and $C \in \mathcal{A}(\Omega)$ such that $\bar{C} \subset B \subset A$. Given $\eta>0$ arbitrary, there exist sequences $\left\{u_{k}\right\}$, $\left\{v_{k}\right\} \subset W^{1, p}(\Omega ; \mathcal{M})$ such that $u_{k}$ and $v_{k}$ converge weakly to $u$ in $W^{1, p}\left(\Omega ; \mathbb{R}^{d}\right), u_{k}(x)=v_{k}(x)=u(x)$ if $\operatorname{dist}(u(x), \mathcal{K})>1$ for a.e. $x \in \Omega, u_{k}$ and $v_{k}$ are uniformly converging to $u$, and

$$
\left\{\begin{array}{l}
\limsup _{k \rightarrow+\infty} \mathcal{F}_{\varepsilon_{k}}\left(u_{k}, B\right) \leq \mathcal{F}_{\mathcal{K}}^{\left\{\varepsilon_{k}\right\}}(u, B)+\eta, \\
\limsup _{k \rightarrow+\infty} \mathcal{F}_{\varepsilon_{k}}\left(v_{k}, A \backslash \bar{C}\right) \leq \mathcal{F}_{\mathcal{K}}^{\left\{\varepsilon_{k}\right\}}(u, A \backslash \bar{C})+\eta
\end{array}\right.
$$

Let $\mathcal{K}^{\prime}:=\{s \in \mathcal{M}: \operatorname{dist}(s, \mathcal{K}) \leq 1\}$, then $\mathcal{K}^{\prime}$ is a compact subset of $\mathcal{M}$ and $u_{k}(x)=v_{k}(x)=u(x)$ if $u(x) \notin \mathcal{K}^{\prime}$ for a.e. $x \in \Omega$.

Consider $L:=\operatorname{dist}(C, \partial B), M \in \mathbb{N}$, and for every $i \in\{0, \ldots, M\}$ define

$$
B_{i}:=\left\{x \in B: \operatorname{dist}(x, \partial B)>\frac{i L}{M}\right\} .
$$

Given $i \in\{0, \ldots, M-1\}$ let $S_{i}:=B_{i} \backslash \overline{B_{i+1}}$, and $\zeta_{i} \in \mathcal{C}_{c}^{\infty}(\Omega ;[0,1])$ be a cut-off function satisfying

$$
\zeta_{i}(x)=\left\{\begin{array}{ll}
1 & \text { in } B_{i+1}, \\
0 & \text { in } \Omega \backslash B_{i},
\end{array} \text { and } \quad\left|\nabla \zeta_{i}\right| \leq \frac{2 M}{L} .\right.
$$


By Lemma 3.2 and Remark 3.3 in [12], there exist $\delta>0, c>0$, and a uniformly continuously differentiable mapping $\Phi: D_{\delta} \times[0,1] \rightarrow \mathcal{M}$, where

$$
D_{\delta}:=\left\{\left(s_{0}, s_{1}\right) \in \mathcal{M} \times \mathcal{M}: \operatorname{dist}\left(s_{0}, \mathcal{K}^{\prime}\right)<\delta, \operatorname{dist}\left(s_{1}, \mathcal{K}^{\prime}\right)<\delta,\left|s_{0}-s_{1}\right|<\delta\right\},
$$

such that

$$
\Phi\left(s_{0}, s_{1}, 0\right)=s_{0}, \quad \Phi\left(s_{0}, s_{1}, 1\right)=s_{1}, \quad \frac{\partial \Phi}{\partial t}\left(s_{0}, s_{1}, t\right) \leq c\left|s_{0}-s_{1}\right|,
$$

and

$$
\left|\Phi\left(s_{0}, s_{1}, t\right)-s_{0}\right| \leq c\left|s_{0}-s_{1}\right| .
$$

Since $\left\{u_{k}\right\}$ and $\left\{v_{k}\right\}$ are uniformly converging to $u$, one can choose $k$ large enough to ensure that

$$
\left\|u_{k}-u\right\|_{L^{\infty}\left(\Omega ; \mathbb{R}^{d}\right)}<\delta, \quad\left\|v_{k}-u\right\|_{L^{\infty}\left(\Omega ; \mathbb{R}^{d}\right)}<\delta \quad \text { and } \quad\left\|u_{k}-v_{k}\right\|_{L^{\infty}\left(\Omega ; \mathbb{R}^{d}\right)}<\delta .
$$

Therefore for a.e. $x \in \Omega$, $\operatorname{dist}\left(u_{k}(x), \mathcal{K}^{\prime}\right)<\delta$ and $\operatorname{dist}\left(v_{k}(x), \mathcal{K}^{\prime}\right)<\delta$ whenever $u(x) \in \mathcal{K}^{\prime}$. Now we are allowed to define

$$
w_{k, i}(x):= \begin{cases}\Phi\left(v_{k}(x), u_{k}(x), \zeta_{i}(x)\right) & \text { if } u(x) \in \mathcal{K}^{\prime} \\ u(x) & \text { if } u(x) \notin \mathcal{K}^{\prime}\end{cases}
$$

and $w_{k, i} \in W^{1, p}(\Omega ; \mathcal{M})$. Using the $p$-growth condition $\left(H_{2}\right)$ together with $(3.4)$, we derive

$$
\begin{aligned}
\int_{A} f\left(\frac{x}{\varepsilon_{k}}, \nabla w_{k, i}\right) \mathrm{d} x \leq \int_{B} f\left(\frac{x}{\varepsilon_{k}}, \nabla u_{k}\right) \mathrm{d} x+\int_{A \backslash \bar{C}} f\left(\frac{x}{\varepsilon_{k}}, \nabla v_{k}\right) \mathrm{d} x & \\
& +C_{0} \int_{S_{i}}\left(1+\left|\nabla u_{k}\right|^{p}+\left|\nabla v_{k}\right|^{p}+M^{p}\left|u_{k}-v_{k}\right|^{p}\right) \mathrm{d} x
\end{aligned}
$$

for some constant $C_{0}>0$ independent of $k, i$ and $M$. Summing up over $i \in\{0, \ldots, M-1\}$ and dividing by $M$ yields

$$
\begin{aligned}
\frac{1}{M} \sum_{i=0}^{M-1} \int_{A} f\left(\frac{x}{\varepsilon_{k}}, \nabla w_{k, i}\right) \mathrm{d} x \leq \int_{B} f\left(\frac{x}{\varepsilon_{k}}, \nabla u_{k}\right) \mathrm{d} x+\int_{A \backslash \bar{C}} f\left(\frac{x}{\varepsilon_{k}}, \nabla v_{k}\right) \mathrm{d} x & \\
& +\frac{C_{0}}{M} \int_{B \backslash \bar{C}}\left(1+\left|\nabla u_{k}\right|^{p}+\left|\nabla v_{k}\right|^{p}+M^{p}\left|u_{k}-v_{k}\right|^{p}\right) \mathrm{d} x .
\end{aligned}
$$

Hence one may find some $i_{k} \in\{0, \ldots, M-1\}$ such that $\bar{w}_{k}:=w_{k, i_{k}}$ satisfies

$$
\begin{aligned}
\int_{A} f\left(\frac{x}{\varepsilon_{k}}, \nabla \bar{w}_{k}\right) \mathrm{d} x \leq \int_{B} f\left(\frac{x}{\varepsilon_{k}}, \nabla u_{k}\right) \mathrm{d} x+\int_{A \backslash \bar{C}} & f\left(\frac{x}{\varepsilon_{k}}, \nabla v_{k}\right) \mathrm{d} x \\
& +\frac{C_{0}}{M} \int_{B \backslash \bar{C}}\left(1+\left|\nabla u_{k}\right|^{p}+\left|\nabla v_{k}\right|^{p}+M^{p}\left|u_{k}-v_{k}\right|^{p}\right) \mathrm{d} x .
\end{aligned}
$$


From (3.4) and (3.5) we deduce that $\bar{w}_{k} \rightarrow u$ uniformly, $\bar{w}_{k} \rightarrow u$ in $W^{1, p}\left(\Omega ; \mathbb{R}^{d}\right)$, and $\bar{w}_{k}(x)=u(x)$ if $\operatorname{dist}(u(x), \mathcal{K})>1$ for a.e. $x \in \Omega$. Taking $\left\{\bar{w}_{k}\right\}$ as competitor for $\mathcal{F}_{\mathcal{K}}^{\left\{\varepsilon_{k}\right\}}(u, A)$, and using (3.6) together with (3.3) leads to

$$
\begin{aligned}
\mathcal{F}_{\mathcal{K}}^{\left\{\varepsilon_{k}\right\}}(u, A) \leq & \limsup _{k \rightarrow+\infty} \mathcal{F}_{\varepsilon_{k}}\left(\bar{w}_{k}, A\right) \\
\leq & \limsup _{k \rightarrow+\infty}\left\{\mathcal{F}_{\varepsilon_{k}}\left(u_{k}, B\right)+\mathcal{F}_{\varepsilon_{k}}\left(v_{k}, A \backslash \bar{C}\right)\right. \\
& \left.+\frac{C_{0}}{M} \int_{B \backslash \bar{C}}\left(1+\left|\nabla u_{k}\right|^{p}+\left|\nabla v_{k}\right|^{p}+M^{p}\left|u_{k}-v_{k}\right|^{p}\right) \mathrm{d} x\right\} \\
\leq & \mathcal{F}_{\mathcal{K}}^{\left\{\varepsilon_{k}\right\}}(u, B)+\mathcal{F}_{\mathcal{K}}^{\left\{\varepsilon_{k}\right\}}(u, A \backslash \bar{C})+2 \eta \\
& +\frac{C_{0}}{M} \sup _{k \in \mathbb{N}} \int_{B \backslash \bar{C}}\left(1+\left|\nabla u_{k}\right|^{p}+\left|\nabla v_{k}\right|^{p}\right) \mathrm{d} x
\end{aligned}
$$

Then property (3.2) arises sending first $M \rightarrow+\infty$, and then $\eta \rightarrow 0$.

Step 2. Now we complete the proof of Lemma 3.1. Using a standard diagonal argument, we construct a subsequence $\left\{\varepsilon_{k}\right\} \searrow 0^{+}$and a sequence $\left\{u_{k}\right\} \subset W^{1, p}(\Omega, \mathcal{M})$ satisfying

$$
\begin{aligned}
\lim _{k \rightarrow+\infty} \mathcal{F}_{\varepsilon_{k}}\left(u_{k}, \Omega\right)=\inf _{\left\{v_{k}\right\}}\left\{\liminf _{k \rightarrow+\infty} \mathcal{F}_{\varepsilon_{k}}\left(v_{k}, \Omega\right): v_{k} \rightarrow u \text { weakly in } W^{1, p}\left(\Omega ; \mathbb{R}^{d}\right),\right. \\
\left.\quad v_{k} \rightarrow u \text { uniformly and } v_{k}(x)=u(x) \text { whenever } \operatorname{dist}(u(x), \mathcal{K})>1 \text { for a.e. } x \in \Omega\right\} .
\end{aligned}
$$

By construction of $\left\{\varepsilon_{k}\right\}$ and $\left\{u_{k}\right\}$, we have $\lim _{k \rightarrow+\infty} \mathcal{F}_{\varepsilon_{k}}\left(u_{k}, \Omega\right)=\mathcal{F}_{\mathcal{K}}^{\left\{\varepsilon_{k}\right\}}(u, \Omega)$. Up to the extraction of a further subsequence, we may assume that

$$
f\left(\frac{\dot{\varepsilon}}{\varepsilon_{k}}, \nabla u_{k}\right) \mathcal{L}^{N}\llcorner\Omega \stackrel{\star}{\rightarrow} \mu \quad \text { in } \mathcal{M}(\Omega)
$$

for some nonnegative Radon measure $\mu \in \mathcal{M}(\Omega)$. By lower semicontinuity, we have

$$
\mu(\Omega) \leq \lim _{k \rightarrow+\infty} \mathcal{F}_{\varepsilon_{k}}\left(u_{k}, \Omega\right)=\mathcal{F}_{\mathcal{K}}^{\left\{\varepsilon_{k}\right\}}(u, \Omega) .
$$

We claim that

$$
\mathcal{F}_{\mathcal{K}}^{\left\{\varepsilon_{k}\right\}}(u, A)=\mu(A) \quad \text { for any } A \in \mathcal{A}(\Omega) .
$$

We fix $A \in \mathcal{A}(\Omega)$ and we start by proving the inequality " $\leq$ ". Given $\eta>0$ arbitrary we can select, in view of (3.1), $C \in \mathcal{A}(\Omega), C \subset \subset A$, such that $\mathcal{F}_{\mathcal{K}}^{\left\{\varepsilon_{k}\right\}}(u, A \backslash \bar{C}) \leq \eta$. Then inequality (3.2) implies that for any $B \in \mathcal{A}(\Omega), C \subset \subset B \subset \subset A$,

$$
\mathcal{F}_{\mathcal{K}}^{\left\{\varepsilon_{k}\right\}}(u, A) \leq \eta+\limsup _{k \rightarrow+\infty} \mathcal{F}_{\varepsilon_{k}}\left(u_{k}, B\right) \leq \eta+\mu(\bar{B}) \leq \eta+\mu(A),
$$

and the conclusion follows from the arbitrariness of $\eta$.

Conversely, for any $B \in \mathcal{A}(\Omega), B \subset \subset A$, we have

$$
\begin{aligned}
\mu(\Omega) \leq \mathcal{F}_{\mathcal{K}}^{\left\{\varepsilon_{k}\right\}}(u, \Omega) & \leq \mathcal{F}_{\mathcal{K}}^{\left\{\varepsilon_{k}\right\}}(u, A)+\mathcal{F}_{\mathcal{K}}^{\left\{\varepsilon_{k}\right\}}(u, \Omega \backslash \bar{B}) \\
& \leq \mathcal{F}_{\mathcal{K}}^{\left\{\varepsilon_{k}\right\}}(u, A)+\mu(\Omega \backslash \bar{B}) \leq \mathcal{F}_{\mathcal{K}}^{\left\{\varepsilon_{k}\right\}}(u, A)+\mu(\Omega \backslash B) \leq \mathcal{F}_{\mathcal{K}}^{\left\{\varepsilon_{k}\right\}}(u, A)+\mu(\Omega)-\mu(B) .
\end{aligned}
$$

Therefore $\mu(B) \leq \mathcal{F}_{\mathcal{K}}^{\left\{\varepsilon_{k}\right\}}(u, A)$ and the conclusion follows by inner regularity of $\mu$. 


\section{The UPPER BOUND}

We now make use of the previous locality result to show the upper bound. This will be done thanks to a blow-up analysis in the spirit of [12], Theorem 3.1.

Lemma 4.1. For every $p \in[1,+\infty)$ and $u \in W^{1, p}(\Omega ; \mathcal{M})$, we have $\mathcal{F}(u) \leq \mathcal{F}_{\text {hom }}(u)$.

Proof. Step 1. Let $u \in W^{1, p}(\Omega ; \mathcal{M})$. Given $R>0$ arbitrary large, we set $\mathcal{K}:=\mathcal{M} \cap B^{d}(0, R)$, and we consider the subsequence $\left\{\varepsilon_{k}\right\}$ given by Lemma 3.1. Obviously $\mathcal{F}(u) \leq \mathcal{F}_{\mathcal{K}}^{\left\{\varepsilon_{k}\right\}}(u, \Omega)$. We claim that

$$
\mathcal{F}_{\mathcal{K}}^{\left\{\varepsilon_{k}\right\}}(u, \Omega) \leq \int_{\Omega}\left\{\chi_{R}(|u|) T f_{\text {hom }}(u, \nabla u)+\beta\left(1-\chi_{R}(|u|)\right)\left(1+|\nabla u|^{p}\right)\right\} \mathrm{d} x
$$

where $\chi_{R}(t)=1$ for $t \leq R$ and $\chi_{R}(t)=0$ otherwise. We postpone the proof of (4.1) to the next step, and we complete now the proof of Lemma 4.1.

Consider a sequence $R_{j} \rightarrow+\infty$ as $j \rightarrow+\infty$. Since $\chi_{R_{j}} \rightarrow 1$ pointwise, we deduce from Fatou's lemma together with (2.4) that

$\mathcal{F}(u) \leq \limsup _{j \rightarrow+\infty} \int_{\Omega}\left\{\chi_{R_{j}}(|u|) T f_{\text {hom }}(u, \nabla u)+\beta\left(1-\chi_{R_{j}}(|u|)\right)\left(1+|\nabla u|^{p}\right)\right\} \mathrm{d} x \leq \int_{\Omega} T f_{\text {hom }}(u, \nabla u) \mathrm{d} x$,

which is the announced estimate.

Step 2. Thanks to Lemma 3.1, to obtain (4.1) it suffices to prove that

$$
\frac{\mathrm{d} \mathcal{F}_{\mathcal{K}}^{\left\{\varepsilon_{k}\right\}}(u, \cdot)}{\mathrm{d} \mathcal{L}^{N}}\left(x_{0}\right) \leq \chi_{R}\left(\left|u\left(x_{0}\right)\right|\right) T f_{\text {hom }}\left(u\left(x_{0}\right), \nabla u\left(x_{0}\right)\right)+\beta\left(1-\chi_{R}\left(\left|u\left(x_{0}\right)\right|\right)\right)\left(1+\left|\nabla u\left(x_{0}\right)\right|^{p}\right)
$$

for $\mathcal{L}^{N}$-a.e. $x_{0} \in \Omega$.

Let $x_{0} \in \Omega$ be a Lebesgue point of $u$ and $\nabla u$ such that $u\left(x_{0}\right) \in \mathcal{M}, \nabla u\left(x_{0}\right) \in\left[T_{u\left(x_{0}\right)}(\mathcal{M})\right]^{N}$, and the Radon-Nikodým derivative of $\mathcal{F}_{\mathcal{K}}^{\left\{\varepsilon_{k}\right\}}(u, \cdot)$ with respect to the Lebesgue measure $\mathcal{L}^{N}$ exists. Note that almost every points in $\Omega$ satisfy these properties. Now set $s_{0}:=u\left(x_{0}\right)$ and $\xi_{0}:=\nabla u\left(x_{0}\right)$.

Case 1. Assume that $s_{0} \notin \mathcal{K}$. Then, using $\left(H_{2}\right)$, we derive that

$$
\begin{aligned}
\frac{\mathrm{d} \mathcal{F}_{\mathcal{K}}^{\left\{\varepsilon_{k}\right\}}(u, \cdot)}{\mathrm{d} \mathcal{L}^{N}}\left(x_{0}\right)=\lim _{\rho \rightarrow 0^{+}} \frac{\mathcal{F}_{\mathcal{K}}^{\left\{\varepsilon_{k}\right\}}\left(u, Q\left(x_{0}, \rho\right)\right)}{\rho^{N}} & \leq \limsup _{\rho \rightarrow 0^{+}} \limsup _{k \rightarrow+\infty} \rho^{-N} \mathcal{F}_{\varepsilon_{k}}\left(u, Q\left(x_{0}, \rho\right)\right) \\
& \leq \lim _{\rho \rightarrow 0^{+}} \frac{\beta}{\rho^{N}} \int_{Q\left(x_{0}, \rho\right)}\left(1+|\nabla u|^{p}\right) \mathrm{d} x=\beta\left(1+\left|\xi_{0}\right|^{p}\right),
\end{aligned}
$$

which is the desired estimate.

Case 2. Now we assume that $s_{0} \in \mathcal{K}$. Fix $0<\eta<1$ arbitrary. By Proposition 2.1, claim (i), there exist $j \in \mathbb{N}$ and $\varphi \in W_{0}^{1, \infty}\left((0, j)^{N} ; T_{s_{0}}(\mathcal{M})\right)$ such that

$$
f_{(0, j)^{N}} f\left(y, \xi_{0}+\nabla \varphi(y)\right) \mathrm{d} y \leq T f_{\text {hom }}\left(s_{0}, \xi_{0}\right)+\eta
$$

Extend $\varphi$ to $\mathbb{R}^{N}$ by $j$-periodicity, and define $\varphi_{k}(x):=\xi_{0} x+\varepsilon_{k} \varphi\left(x / \varepsilon_{k}\right)$. 
Let $\mathcal{U}$ be an open neighborhood of $\mathcal{M}$ such that the nearest point projection $\Pi: \mathcal{U} \rightarrow \mathcal{M}$ defines a $\mathcal{C}^{1}$-mapping. Fix $\sigma, \delta_{0} \in(0,1)$ such that $B^{d}\left(s_{0}, 2 \delta_{0}\right) \subset \mathcal{U}$, and consider $\delta=\delta(\sigma) \in\left(0, \delta_{0}\right)$ for which

$$
\left|\nabla \Pi(s)-\nabla \Pi\left(s^{\prime}\right)\right|<\sigma \quad \text { for all } s, s^{\prime} \in B^{d}\left(s_{0}, \delta_{0}\right) \text { satisfying }\left|s-s^{\prime}\right|<\delta .
$$

Next we introduce a cut-off function $\zeta \in \mathcal{C}_{c}^{\infty}\left(\mathbb{R}^{d} ;[0,1]\right)$ satisfying

$$
\zeta(x)=\left\{\begin{array}{ll}
1 & \text { for } x \in B^{d}(0, \delta / 4), \\
0 & \text { for } x \notin B^{d}(0, \delta / 2),
\end{array} \quad \text { with } \quad|\nabla \zeta| \leq \frac{C}{\delta},\right.
$$

and we define

Let $k_{0} \in \mathbb{N}$ be such that

$$
w_{k}(x):=u(x)+\varepsilon_{k} \zeta\left(u(x)-s_{0}\right) \varphi\left(x / \varepsilon_{k}\right) .
$$

$$
\max \left\{\varepsilon_{k}\|\varphi\|_{L^{\infty}\left((0, j)^{N} ; \mathbb{R}^{d}\right)}\|\nabla \zeta\|_{L^{\infty}\left(\mathbb{R}^{d} ; \mathbb{R}^{d}\right)}, \frac{2 \varepsilon_{k}\|\varphi\|_{L^{\infty}\left((0, j)^{N} ; \mathbb{R}^{d}\right)}}{\delta}\right\}<1 \quad \text { for any } k \geq k_{0} .
$$

Define for every $k \geq k_{0}$,

$$
u_{k}(x):=\Pi\left(w_{k}(x)\right) .
$$

Remark that by (4.4), for a.e. $x \in \Omega$ and all $k \geq k_{0}$, one has $w_{k}(x) \in B^{d}\left(s_{0}, \delta\right)$ whenever $\left|u(x)-s_{0}\right|<\delta / 2$ while $w_{k}(x)=u(x)$ when $\left|u(x)-s_{0}\right| \geq \delta / 2$. Hence $u_{k}$ is well defined, $\left\{u_{k}\right\} \subset W^{1, p}(\Omega ; \mathcal{M})$, and for a.e. $x \in \Omega$, $u_{k}(x)=u(x)$ whenever $\operatorname{dist}(u(x), \mathcal{K})>1$. Moreover,

$$
\left\|u_{k}-u\right\|_{L^{\infty}\left(\Omega ; \mathbb{R}^{d}\right)}=\left\|\Pi\left(w_{k}\right)-\Pi(u)\right\|_{L^{\infty}\left(\left\{\left|u-s_{0}\right|<\delta / 2\right\} ; \mathbb{R}^{d}\right)} \leq \varepsilon_{k}\|\nabla \Pi\|_{L^{\infty}\left(B^{d}\left(s_{0}, \delta_{0}\right) ; \mathbb{R}^{d}\right)}\|\varphi\|_{L^{\infty}\left((0, j)^{N} ; \mathbb{R}^{d}\right)} \rightarrow 0
$$

as $k \rightarrow+\infty$. Now the Chain Rule formula yields

$$
\nabla u_{k}(x)=\nabla \Pi\left(w_{k}(x)\right)\left(\nabla u(x)+\varepsilon_{k}\left(\varphi\left(x / \varepsilon_{k}\right) \otimes \nabla \zeta\left(u(x)-s_{0}\right)\right) \nabla u(x)+\zeta\left(u(x)-s_{0}\right) \nabla \varphi\left(x / \varepsilon_{k}\right)\right),
$$

and consequently

$$
\left|\nabla u_{k}(x)\right| \leq\|\nabla \Pi\|_{L^{\infty}\left(B^{d}\left(s_{0}, \delta_{0}\right) ; \mathbb{R}^{d}\right)}\left(\left(1+\varepsilon_{k}\|\varphi\|_{L^{\infty}\left((0, j)^{N} ; \mathbb{R}^{d}\right)}\|\nabla \zeta\|_{L^{\infty}\left(\mathbb{R}^{d} ; \mathbb{R}^{d}\right)}\right)|\nabla u(x)|+\|\nabla \varphi\|_{L^{\infty}\left((0, j)^{N} ; \mathbb{R}^{d \times N}\right)}\right) .
$$

By (4.4) it follows that for any $k \geq k_{0}$,

$$
\left|\nabla u_{k}(x)\right| \leq C_{0}\left(\left|\nabla u(x)-\xi_{0}\right|+1\right)
$$

for some constant $C_{0}=C_{0}\left(s_{0}, \xi_{0}, \delta_{0}, \eta\right)>0$ independent of $x$ and $k$. Hence the sequence $\left\{u_{k}\right\}$ is uniformly bounded in $W^{1, p}\left(\Omega ; \mathbb{R}^{d}\right)$ so that $u_{k} \rightarrow u$ in $W^{1, p}\left(\Omega ; \mathbb{R}^{d}\right)$.

Then we observe that $\left|\nabla u_{k}\right| \leq 2 C_{0}$ a.e. in $\left\{\left|\nabla u-\xi_{0}\right|<\sigma\right\}$ while

$$
\left\|\nabla \varphi_{k}\right\|_{L^{\infty}\left(\Omega ; \mathbb{R}^{d \times N}\right)} \leq\left|\xi_{0}\right|+\|\nabla \varphi\|_{L^{\infty}\left((0, j)^{N} ; \mathbb{R}^{d \times N}\right)} .
$$

Set

$$
M:=\max \left\{2 C_{0},\left|\xi_{0}\right|+\|\nabla \varphi\|_{L^{\infty}\left((0, j)^{N} ; \mathbb{R}^{d \times N}\right)}\right\},
$$

(which only depends on $s_{0}, \xi_{0}, \delta_{0}$ and $\eta$ ) so that

$$
\left|\nabla u_{k}\right| \leq M \quad \text { and } \quad\left|\nabla \varphi_{k}\right| \leq M \quad \text { a.e. in }\left\{\left|\nabla u-\xi_{0}\right|<\sigma\right\} .
$$


Next for a.e. $x \in\left\{\left|u-s_{0}\right|<\delta / 4\right\} \cap\left\{\left|\nabla u-\xi_{0}\right|<\sigma\right\}$, we have $\zeta\left(u(x)-s_{0}\right)=1$ and

$$
\begin{aligned}
\left|\nabla u_{k}(x)-\nabla \varphi_{k}(x)\right| \leq & \left|\nabla \Pi\left(w_{k}\right) \nabla u(x)-\xi_{0}\right|+\left|\nabla \Pi\left(w_{k}\right) \nabla \varphi\left(x / \varepsilon_{k}\right)-\nabla \varphi\left(x / \varepsilon_{k}\right)\right| \\
\leq & \left|\nabla \Pi\left(w_{k}\right)-\nabla \Pi\left(s_{0}\right)\right||\nabla u(x)|+\left|\nabla \Pi\left(s_{0}\right)\right|\left|\nabla u(x)-\xi_{0}\right| \\
& +\left|\nabla \Pi\left(w_{k}\right)-\nabla \Pi\left(s_{0}\right)\right| \mid \nabla \varphi \|_{L^{\infty}\left((0, j)^{N} ; \mathbb{R}^{d \times N}\right)},
\end{aligned}
$$

where, in the last inequality, we have used the fact that $\nabla \Pi\left(s_{0}\right) \nabla \varphi(y)=\nabla \varphi(y)$ since $\nabla \varphi(y) \in\left[T_{s_{0}}(\mathcal{M})\right]^{N}$ for a.e. $y \in \mathbb{R}^{N}$. Using (4.3) and the fact that $\left|w_{k}-s_{0}\right|<\delta$ a.e. in $\left\{\left|u-s_{0}\right|<\delta / 4\right\} \cap\left\{\left|\nabla u-\xi_{0}\right|<\sigma\right\}$, we deduce

$$
\left|\nabla u_{k}(x)-\nabla \varphi_{k}(x)\right| \leq\left(|\nabla u(x)|+\left|\nabla \Pi\left(s_{0}\right)\right|+\|\nabla \varphi\|_{L^{\infty}\left((0, j)^{N} ; \mathbb{R}^{d \times N}\right)}\right) \sigma \leq C_{1} \sigma
$$

for a.e. $x \in\left\{\left|u-s_{0}\right|<\delta / 4\right\} \cap\left\{\left|\nabla u-\xi_{0}\right|<\sigma\right\}$, where $C_{1}=C_{1}\left(s_{0}, \xi_{0}, \delta_{0}, \eta\right)>0$ is a constant independent of $\sigma$, $k$ and $x$.

Now we estimate

$$
\begin{aligned}
& \frac{\mathrm{d} \mathcal{F}_{\mathcal{K}}^{\left\{\varepsilon_{k}\right\}}(u, \cdot)}{\mathrm{d} \mathcal{L}^{N}}\left(x_{0}\right)=\lim _{\rho \rightarrow 0^{+}} \frac{\mathcal{F}_{\mathcal{K}}^{\left\{\varepsilon_{k}\right\}}\left(u, Q\left(x_{0}, \rho\right)\right)}{\rho^{N}} \\
& \leq \limsup _{\rho \rightarrow 0^{+}} \limsup _{k \rightarrow+\infty} \frac{1}{\rho^{N}} \int_{Q\left(x_{0}, \rho\right)} f\left(\frac{x}{\varepsilon_{k}}, \nabla u_{k}\right) \mathrm{d} x \\
& \leq \limsup _{\rho \rightarrow 0^{+}} \limsup _{k \rightarrow+\infty} \frac{1}{\rho^{N}} \int_{Q\left(x_{0}, \rho\right) \cap\left\{\left|u-s_{0}\right| \geq \delta / 4\right\}} f\left(\frac{x}{\varepsilon_{k}}, \nabla u_{k}\right) \mathrm{d} x \\
& +\limsup _{\rho \rightarrow 0^{+}} \limsup _{k \rightarrow+\infty} \frac{1}{\rho^{N}} \int_{Q\left(x_{0}, \rho\right) \cap\left\{\left|u-s_{0}\right|<\delta / 4\right\} \cap\left\{\left|\nabla u-\xi_{0}\right|<\sigma\right\}} f\left(\frac{x}{\varepsilon_{k}}, \nabla u_{k}\right) \mathrm{d} x \\
& +\limsup _{\rho \rightarrow 0^{+}} \limsup _{k \rightarrow+\infty} \frac{1}{\rho^{N}} \int_{Q\left(x_{0}, \rho\right) \cap\left\{\left|u-s_{0}\right|<\delta / 4\right\} \cap\left\{\left|\nabla u-\xi_{0}\right| \geq \sigma\right\}} f\left(\frac{x}{\varepsilon_{k}}, \nabla u_{k}\right) \mathrm{d} x \\
& =: I_{1}+I_{2}+I_{3} \text {. }
\end{aligned}
$$

Thanks to (4.5), the $p$-growth condition $\left(H_{2}\right)$ and our choice of $x_{0}$, we have

$$
\begin{aligned}
I_{1} & \leq C \limsup _{\rho \rightarrow 0^{+}} \frac{1}{\rho^{N}} \int_{Q\left(x_{0}, \rho\right) \cap\left\{\left|u-s_{0}\right| \geq \delta / 4\right\}}\left(1+\left|\nabla u(x)-\xi_{0}\right|^{p}\right) \mathrm{d} x \\
& \leq C \limsup _{\rho \rightarrow 0^{+}} f_{Q\left(x_{0}, \rho\right)}\left|\nabla u(x)-\xi_{0}\right|^{p} \mathrm{~d} x+\frac{4 C}{\delta} \limsup _{\rho \rightarrow 0^{+}} f_{Q\left(x_{0}, \rho\right)}\left|u(x)-s_{0}\right| \mathrm{d} x=0,
\end{aligned}
$$

while

$$
\begin{aligned}
I_{3} & \leq C \limsup _{\rho \rightarrow 0^{+}} \frac{1}{\rho^{N}} \int_{Q\left(x_{0}, \rho\right) \cap\left\{\left|u-s_{0}\right|<\delta / 4\right\} \cap\left\{\left|\nabla u-\xi_{0}\right| \geq \sigma\right\}}\left(1+\left|\nabla u(x)-\xi_{0}\right|^{p}\right) \mathrm{d} x \\
& \leq C \limsup _{\rho \rightarrow 0^{+}} f_{Q\left(x_{0}, \rho\right)}\left|\nabla u(x)-\xi_{0}\right|^{p} \mathrm{~d} x+\frac{C}{\sigma} \limsup _{\rho \rightarrow 0^{+}} f_{Q\left(x_{0}, \rho\right)}\left|\nabla u(x)-\xi_{0}\right| \mathrm{d} x=0 .
\end{aligned}
$$

Let us now treat the integral $I_{2}$. Since, for a.e. $y \in \mathbb{R}^{N}$, the function $f(y, \cdot)$ is continuous, it is uniformly continuous on $B^{d \times N}(0, M)$ where $M>0$ is given in (4.6). Define the modulus of continuity of $f(y, \cdot)$ over $B^{d \times N}(0, M)$ by

$$
\omega(y, t):=\sup \left\{\left|f(y, \xi)-f\left(y, \xi^{\prime}\right)\right|: \xi, \xi^{\prime} \in B^{d \times N}(0, M) \text { and }\left|\xi-\xi^{\prime}\right| \leq t\right\}
$$


It turns out that $\omega(y, \cdot)$ is increasing, continuous and $\omega(y, 0)=0$, while $\omega(\cdot, t)$ is measurable (since the supremum can be restricted to all admissible $\xi$ and $\xi^{\prime}$ having rational entries) and 1-periodic. Thanks to (4.7) and (4.8) we get that

$$
\left|f\left(\frac{x}{\varepsilon_{k}}, \nabla u_{k}(x)\right)-f\left(\frac{x}{\varepsilon_{k}}, \nabla \varphi_{k}(x)\right)\right| \leq \omega\left(\frac{x}{\varepsilon_{k}}, C_{1} \sigma\right)
$$

for a.e. $x \in Q\left(x_{0}, \rho\right) \cap\left\{\left|u-s_{0}\right|<\delta / 4\right\} \cap\left\{\left|\nabla u-\xi_{0}\right|<\sigma\right\}$.

Integrating over the set $Q\left(x_{0}, \rho\right) \cap\left\{\left|u-s_{0}\right|<\delta / 4\right\} \cap\left\{\left|\nabla u-\xi_{0}\right|<\sigma\right\}$, and taking the limit as $k \rightarrow+\infty$, we obtain in view of the Riemann-Lebesgue lemma that

$$
\begin{aligned}
\limsup _{k \rightarrow+\infty} \rho^{-N} \int_{Q\left(x_{0}, \rho\right) \cap\left\{\left|u-s_{0}\right|<\delta / 4\right\} \cap\left\{\left|\nabla u-\xi_{0}\right|<\sigma\right\}}\left|f\left(\frac{x}{\varepsilon_{k}}, \nabla u_{k}(x)\right)-f\left(\frac{x}{\varepsilon_{k}}, \nabla \varphi_{k}(x)\right)\right| \mathrm{d} x \leq \\
\limsup _{k \rightarrow+\infty} \rho^{-N} \int_{Q\left(x_{0}, \rho\right)} \omega\left(\frac{x}{\varepsilon_{k}}, C_{1} \sigma\right) \mathrm{d} x=\int_{Q} \omega\left(y, C_{1} \sigma\right) \mathrm{d} y,
\end{aligned}
$$

where we have used the fact that $y \mapsto \omega\left(y, C_{1} \sigma\right)$ is a measurable 1-periodic function. Observe that the Dominated Convergence Theorem together with $\omega(y, 0)=0$ implies

$$
\lim _{\sigma \rightarrow 0^{+}} \int_{Q} \omega\left(y, C_{1} \sigma\right) \mathrm{d} y=0 .
$$

We have obtained

$$
I_{2} \leq \limsup _{\rho \rightarrow 0^{+}} \limsup _{k \rightarrow+\infty} \frac{1}{\rho^{N}} \int_{Q\left(x_{0}, \rho\right)} f\left(\frac{x}{\varepsilon_{k}}, \nabla \varphi_{k}\right) \mathrm{d} x+\int_{Q} \omega\left(y, C_{1} \sigma\right) \mathrm{d} y .
$$

Using the definition of $\varphi_{k}$ and the Riemann-Lebesgue lemma, we infer from (4.2) that

$$
\underset{\rho \rightarrow 0^{+}}{\limsup } \limsup _{k \rightarrow+\infty} \frac{1}{\rho^{N}} \int_{Q\left(x_{0}, \rho\right)} f\left(\frac{x}{\varepsilon_{k}}, \xi_{0}+\nabla \varphi\left(\frac{x}{\varepsilon_{k}}\right)\right) \mathrm{d} x=f_{(0, j)^{N}} f\left(y, \xi_{0}+\nabla \varphi(y)\right) \mathrm{d} y \leq T f_{\text {hom }}\left(s_{0}, \xi_{0}\right)+\eta .
$$

Hence gathering (4.9)-(4.11), (4.13) and (4.14) we deduce that

$$
\frac{\mathrm{d} \mathcal{F}_{\mathcal{K}}^{\left\{\varepsilon_{k}\right\}}(u, \cdot)}{\mathrm{d} \mathcal{L}^{N}}\left(x_{0}\right) \leq T f_{\text {hom }}\left(s_{0}, \xi_{0}\right)+\int_{Q} \omega\left(y, C_{1} \sigma\right) \mathrm{d} y+\eta
$$

Thanks to (4.12), the thesis follows sending first $\sigma \rightarrow 0$, and then $\eta \rightarrow 0$.

\section{THE LOWER BOUND}

We now investigate the $\Gamma$-lim inf inequality still through the blow-up method. In contrast with Lemma 4.1 we will distinguish energies with superlinear growth and energies with linear growth. We will conclude this section with the proofs of Theorems 1.1 and 1.2.

\subsection{The case of superlinear growth}

The case $p>1$ is based on an equi-integrability result known as Decomposition Lemma [17], Lemma 1.2, which allows to consider sequences with $p$-equi-integrable gradients. It enables to use properties valid up to sets where the energy remains small. 
Lemma 5.1. Assume $p \in(1,+\infty)$. Then $\mathcal{F}(u) \geq \mathcal{F}_{\text {hom }}(u)$ for every $u \in W^{1, p}(\Omega ; \mathcal{M})$.

Proof. Let $u \in W^{1, p}(\Omega ; \mathcal{M})$. By a standard diagonal argument, we first obtain a subsequence $\left\{\varepsilon_{n}\right\}$ (not relabeled) and $\left\{u_{n}\right\} \subset W^{1, p}(\Omega ; \mathcal{M})$ such that $u_{n} \rightarrow u$ in $L^{p}\left(\Omega ; \mathbb{R}^{d}\right)$ and

$$
\mathcal{F}(u)=\lim _{n \rightarrow+\infty} \int_{\Omega} f\left(\frac{x}{\varepsilon_{n}}, \nabla u_{n}\right) \mathrm{d} x<+\infty .
$$

Define the sequence of nonnegative Radon measures

$$
\mu_{n}:=f\left(\frac{\cdot}{\varepsilon_{n}}, \nabla u_{n}\right) \mathcal{L}^{N}\llcorner\Omega
$$

Extracting a further subsequence if necessary, we may assume that there exists a nonnegative Radon measure $\mu \in \mathcal{M}(\Omega)$ such that $\mu_{n} \stackrel{*}{\rightarrow} \mu$ in $\mathcal{M}(\Omega)$. Using Lebesgue Differentiation Theorem one can split $\mu$ into the sum of two mutually disjoint nonnegative measures $\mu=\mu^{a}+\mu^{s}$ where $\mu^{a} \ll \mathcal{L}^{N}$ and $\mu^{s}$ is singular with respect to $\mathcal{L}^{N}$. Since $\mu^{a}(\Omega) \leq \mu(\Omega) \leq \mathcal{F}(u)$, it is enough to check that

$$
\frac{\mathrm{d} \mu}{\mathrm{d} \mathcal{L}^{N}}\left(x_{0}\right) \geq T f_{\text {hom }}\left(u\left(x_{0}\right), \nabla u\left(x_{0}\right)\right) \quad \text { for } \mathcal{L}^{N} \text {-a.e. } x_{0} \in \Omega
$$

Step 1. Select a point $x_{0} \in \Omega$ which is a Lebesgue point of $u$ and $\nabla u$, a point of approximate differentiability of $u$ (so that $u\left(x_{0}\right) \in \mathcal{M}, \nabla u\left(x_{0}\right) \in\left[T_{u\left(x_{0}\right)}(\mathcal{M})\right]^{N}$ ), and such that the Radon-Nikodým derivative of $\mu$ with respect to the Lebesgue measure $\mathcal{L}^{N}$ exists and is finite. Note that almost every points $x_{0}$ in $\Omega$ satisfy these properties. As in the proof of Lemma 4.1, set $s_{0}:=u\left(x_{0}\right)$ and $\xi_{0}:=\nabla u\left(x_{0}\right)$.

Let $\left\{\rho_{k}\right\} \searrow 0^{+}$be such that $\mu\left(\partial Q\left(x_{0}, \rho_{k}\right)\right)=0$ for every $k \in \mathbb{N}$. Using the integrand $\bar{f}$ defined in $(2.1)$ one obtains

$$
\begin{aligned}
\frac{\mathrm{d} \mu}{\mathrm{d} \mathcal{L}^{N}}\left(x_{0}\right) & =\lim _{k \rightarrow+\infty} \frac{\mu\left(Q\left(x_{0}, \rho_{k}\right)\right)}{\rho_{k}^{N}} \\
& =\lim _{k \rightarrow+\infty} \lim _{n \rightarrow+\infty} \frac{\mu_{n}\left(Q\left(x_{0}, \rho_{k}\right)\right)}{\rho_{k}^{N}} \\
& =\lim _{k \rightarrow+\infty} \lim _{n \rightarrow+\infty} \int_{Q} f\left(\frac{x_{0}+\rho_{k} y}{\varepsilon_{n}}, \nabla u_{n}\left(x_{0}+\rho_{k} y\right)\right) \mathrm{d} y \\
& =\lim _{k \rightarrow+\infty} \lim _{n \rightarrow+\infty} \int_{Q} \bar{f}\left(\frac{x_{0}+\rho_{k} y}{\varepsilon_{n}}, u_{n}\left(x_{0}+\rho_{k} y\right), \nabla u_{n}\left(x_{0}+\rho_{k} y\right)\right) \mathrm{d} y \\
& =\lim _{k \rightarrow+\infty} \lim _{n \rightarrow+\infty} \int_{Q} \bar{f}\left(\frac{x_{0}+\rho_{k} y}{\varepsilon_{n}}, s_{0}+\rho_{k} v_{n, k}(y), \nabla v_{n, k}(y)\right) \mathrm{d} y,
\end{aligned}
$$

where we have set $v_{n, k}(y):=\left[u_{n}\left(x_{0}+\rho_{k} y\right)-s_{0}\right] / \rho_{k}$. Note that since $x_{0}$ is a point of approximate differentiability of $u$ and $u_{n} \rightarrow u$ in $L^{p}\left(\Omega ; \mathbb{R}^{d}\right)$, we have

$$
\lim _{k \rightarrow+\infty} \lim _{n \rightarrow+\infty} \int_{Q}\left|v_{n, k}(y)-\xi_{0} y\right|^{p} \mathrm{~d} y=\lim _{k \rightarrow+\infty} \int_{Q\left(x_{0}, \rho_{k}\right)} \frac{\left|u(y)-s_{0}-\xi_{0}\left(y-x_{0}\right)\right|^{p}}{\rho_{k}^{N+p}} \mathrm{~d} y=0 .
$$

Hence one can find a diagonal sequence $\varepsilon_{k}:=\varepsilon_{n_{k}}<\rho_{k}^{2}$ such that, setting $v_{k}(y):=v_{n_{k}, k}(y)$ and $v_{0}(y):=\xi_{0} y$, $v_{k} \rightarrow v_{0}$ in $L^{p}\left(Q ; \mathbb{R}^{d}\right)$ and

$$
\frac{\mathrm{d} \mu}{\mathrm{d} \mathcal{L}^{N}}\left(x_{0}\right)=\lim _{k \rightarrow+\infty} \int_{Q} \bar{f}\left(\frac{x_{0}+\rho_{k} y}{\varepsilon_{k}}, s_{0}+\rho_{k} v_{k}(y), \nabla v_{k}(y)\right) \mathrm{d} y
$$


Next observe that $\left\{\nabla v_{k}\right\}$ is bounded in $L^{p}\left(Q ; \mathbb{R}^{d \times N}\right)$ thanks to the coercivity condition (2.2). By the Decomposition Lemma [17], Lemma 1.2, we now find a sequence $\left\{\bar{v}_{k}\right\} \subset W^{1, \infty}\left(Q ; \mathbb{R}^{d}\right)$ such that $\bar{v}_{k}=v_{0}$ on a neighborhood of $\partial Q, \bar{v}_{k} \rightarrow v_{0}$ in $L^{p}\left(Q ; \mathbb{R}^{d}\right)$, the sequence of gradients $\left\{\left|\nabla \bar{v}_{k}\right|^{p}\right\}$ is equi-integrable, and

$$
\lim _{k \rightarrow+\infty} \int_{Q} \bar{f}\left(\frac{x_{0}+\rho_{k} y}{\varepsilon_{k}}, s_{0}+\rho_{k} v_{k}(y), \nabla v_{k}(y)\right) \mathrm{d} y \geq \limsup _{k \rightarrow+\infty} \int_{Q} \bar{f}\left(\frac{x_{0}+\rho_{k} y}{\varepsilon_{k}}, s_{0}+\rho_{k} v_{k}(y), \nabla \bar{v}_{k}(y)\right) \mathrm{d} y .
$$

Step 2. Write

and define

$$
\frac{x_{0}}{\varepsilon_{k}}=m_{k}+s_{k} \quad \text { with } m_{k} \in \mathbb{Z}^{N} \text { and } s_{k} \in[0,1)^{N},
$$

$$
x_{k}:=\frac{\varepsilon_{k}}{\rho_{k}} s_{k} \rightarrow 0 \quad \text { and } \quad \delta_{k}:=\varepsilon_{k} / \rho_{k} \rightarrow 0 .
$$

By the 1-periodicity of $\bar{f}$ with respect to its first variable, (5.1) and (5.2), we infer

$$
\begin{aligned}
\frac{\mathrm{d} \mu}{\mathrm{d} \mathcal{L}^{N}}\left(x_{0}\right) & \geq \limsup _{k \rightarrow+\infty} \int_{Q} \bar{f}\left(\frac{x_{k}+y}{\delta_{k}}, s_{0}+\rho_{k} v_{k}(y), \nabla \bar{v}_{k}(y)\right) \mathrm{d} y \\
& \geq \limsup _{k \rightarrow+\infty} \int_{x_{k}+Q} \bar{f}\left(\frac{y}{\delta_{k}}, s_{0}+\rho_{k} v_{k}\left(y-x_{k}\right), \nabla \bar{v}_{k}\left(y-x_{k}\right)\right) \mathrm{d} y .
\end{aligned}
$$

Extend $v_{k}$ by 0 , and $\bar{v}_{k}$ by $v_{0}$ to the whole $\mathbb{R}^{N}$. As $x_{k} \rightarrow 0$ it follows that $\mathcal{L}^{N}\left(\left(Q-x_{k}\right) \triangle Q\right) \rightarrow 0$, and the equi-integrability of $\left\{\left|\nabla \bar{v}_{k}\right|^{p}\right\}$ together with the $p$-growth condition (2.2) implies

$$
\int_{Q \triangle\left(x_{k}+Q\right)} \bar{f}\left(\frac{y}{\delta_{k}}, s_{0}+\rho_{k} v_{k}\left(y-x_{k}\right), \nabla \bar{v}_{k}\left(y-x_{k}\right)\right) \mathrm{d} y \leq \beta^{\prime} \int_{\left(Q-x_{k}\right) \triangle Q}\left(1+\left|\nabla \bar{v}_{k}\right|^{p}\right) \mathrm{d} y \rightarrow 0 .
$$

Hence (5.3) yields

$$
\frac{\mathrm{d} \mu}{\mathrm{d} \mathcal{L}^{N}}\left(x_{0}\right) \geq \limsup _{k \rightarrow+\infty} \int_{Q} \bar{f}\left(\frac{y}{\delta_{k}}, s_{0}+\rho_{k} w_{k}, \nabla \bar{w}_{k}\right) \mathrm{d} y,
$$

where $w_{k}(y):=v_{k}\left(y-x_{k}\right)$ and $\bar{w}_{k}(y):=\bar{v}_{k}\left(y-x_{k}\right)$ converge to $v_{0}$ in $L^{p}\left(Q ; \mathbb{R}^{d}\right)$, and $\left\{\left|\nabla \bar{w}_{k}\right|^{p}\right\}$ is equi-integrable as well.

Step 3. For $M>1$ and $k \in \mathbb{N}$, consider the set $E_{M, k}:=\left\{x \in Q:\left|\nabla \bar{w}_{k}\right| \leq M\right\}$. By Chebyschev inequality, (5.4) and $(2.2), \mathcal{L}^{N}\left(Q \backslash E_{M, k}\right) \leq C / M^{p}$ for some constant $C>0$ independent of $k$ and $M$.

By the Scorza-Dragoni Theorem (see [14], p. 235), for any $\eta>0$, we may find a compact set $K_{\eta} \subset \bar{Q}$ such that $\mathcal{L}^{N}\left(\bar{Q} \backslash K_{\eta}\right)<\eta$ and $f: K_{\eta} \times \mathbb{R}^{d \times N} \rightarrow[0,+\infty)$ is continuous. In particular the restriction of $\bar{f}(\cdot, s, \cdot)$ to $K_{\eta} \times B^{d \times N}(0, M)$ is uniformly continuous for every $s \in \mathcal{M}$. Therefore the function $\Psi_{\eta, M}:[0,+\infty) \rightarrow[0,+\infty)$ defined by

$$
\Psi_{\eta, M}(t)=\sup \left\{\left|f(y, \xi)-f\left(y, \xi^{\prime}\right)\right|: y \in K_{\eta}, \xi, \xi^{\prime} \in B^{d \times N}(0, M),\left|\xi-\xi^{\prime}\right| \leq t\right\},
$$

is continuous, satisfies $\Psi_{\eta, M}(0)=0$, and is bounded. In view of (2.1), we have

$$
\left|\bar{f}\left(y, s_{1}, \xi\right)-\bar{f}\left(y, s_{2}, \xi\right)\right| \leq \Psi_{\eta, M}\left(M\left|\mathbf{P}_{s_{1}}-\mathbf{P}_{s_{2}}\right|\right)+C_{M}\left|\mathbf{P}_{s_{1}}-\mathbf{P}_{s_{2}}\right|=: \tilde{\Psi}_{\eta, M}\left(\left|\mathbf{P}_{s_{1}}-\mathbf{P}_{s_{2}}\right|\right)
$$

for every $y \in K_{\eta}, s_{1}, s_{2} \in \mathcal{M}$ and $\xi \in B^{d \times N}(0, M)$, where the constant $C_{M}>0$ only depends on $M$ and $p$. Define

$$
K_{\eta}^{\text {per }}:=\bigcup_{\ell \in \mathbb{Z}^{N}}\left(\ell+K_{\eta}\right)
$$

Since $\bar{f}$ is 1-periodic in the first variable,

$$
\left|\bar{f}\left(y, s_{1}, \xi\right)-\bar{f}\left(y, s_{2}, \xi\right)\right| \leq \tilde{\Psi}_{\eta, M}\left(\left|\mathbf{P}_{s_{1}}-\mathbf{P}_{s_{2}}\right|\right)
$$


for every $y \in K_{\eta}^{\text {per }}, s_{1}, s_{2} \in \mathcal{M}$ and $\xi \in B^{d \times N}(0, M)$. From (5.4) and (5.5) it follows that

$$
\begin{aligned}
\frac{\mathrm{d} \mu}{\mathrm{d} \mathcal{L}^{N}}\left(x_{0}\right) \geq & \limsup _{k \rightarrow+\infty} \int_{E_{M, k} \cap\left(\delta_{k} K_{\eta}^{\text {per }}\right)} \bar{f}\left(\frac{y}{\delta_{k}}, s_{0}+\rho_{k} w_{k}, \nabla \bar{w}_{k}\right) \mathrm{d} y \\
\geq & \limsup _{k \rightarrow+\infty} \int_{E_{M, k} \cap\left(\delta_{k} K_{\eta}^{\text {per }}\right)} \bar{f}\left(\frac{y}{\delta_{k}}, s_{0}, \nabla \bar{w}_{k}\right) \mathrm{d} y \\
& -\limsup _{k \rightarrow+\infty} \int_{Q} \tilde{\Psi}_{\eta, M}\left(\left|\mathbf{P}_{s_{0}+\rho_{k} w_{k}(y)}-\mathbf{P}_{s_{0}}\right|\right) \mathrm{d} y
\end{aligned}
$$

Since $\tilde{\Psi}_{\eta, M}$ is continuous and bounded, $\tilde{\Psi}_{\eta, M}(0)=0$, and (up to a subsequence) $\mathbf{P}_{s_{0}+\rho_{k} w_{k}(y)} \rightarrow \mathbf{P}_{s_{0}}$ for a.e. $y \in Q$, we obtain by Dominated Convergence that

$$
\lim _{k \rightarrow+\infty} \int_{Q} \tilde{\Psi}_{\eta, M}\left(\left|\mathbf{P}_{s_{0}+\rho_{k} w_{k}(y)}-\mathbf{P}_{s_{0}}\right|\right) \mathrm{d} y=0
$$

and thus

$$
\frac{\mathrm{d} \mu}{\mathrm{d} \mathcal{L}^{N}}\left(x_{0}\right) \geq \limsup _{k \rightarrow+\infty} \int_{E_{M, k} \cap\left(\delta_{k} K_{\eta}^{\text {per }}\right)} \bar{f}\left(\frac{y}{\delta_{k}}, s_{0}, \nabla \bar{w}_{k}\right) \mathrm{d} y .
$$

From the $p$-growth condition (2.2) and the Riemann-Lebesgue Lemma, we deduce that

$$
\begin{aligned}
\limsup _{k \rightarrow+\infty} \int_{E_{M, k} \backslash\left(\delta_{k} K_{\eta}^{\text {per }}\right)} \bar{f}\left(\frac{y}{\delta_{k}}, s_{0}, \nabla \bar{w}_{k}\right) \mathrm{d} y & \leq \limsup _{k \rightarrow+\infty} \beta^{\prime}\left(1+M^{p}\right) \mathcal{L}^{N}\left(Q \backslash\left(\delta_{k} K_{\eta}^{\text {per }}\right)\right) \\
& =\beta^{\prime}\left(1+M^{p}\right) \mathcal{L}^{N}\left(Q \backslash K_{\eta}\right) \leq \beta^{\prime}\left(1+M^{p}\right) \eta .
\end{aligned}
$$

Hence (5.6) yields

$$
\frac{\mathrm{d} \mu}{\mathrm{d} \mathcal{L}^{N}}\left(x_{0}\right) \geq \limsup _{k \rightarrow+\infty} \int_{E_{M, k}} \bar{f}\left(\frac{y}{\delta_{k}}, s_{0}, \nabla \bar{w}_{k}\right) \mathrm{d} y-\beta^{\prime}\left(1+M^{p}\right) \eta,
$$

and sending $\eta \rightarrow 0$, we derive

$$
\frac{\mathrm{d} \mu}{\mathrm{d} \mathcal{L}^{N}}\left(x_{0}\right) \geq \limsup _{k \rightarrow+\infty} \int_{E_{M, k}} \bar{f}\left(\frac{y}{\delta_{k}}, s_{0}, \nabla \bar{w}_{k}\right) \mathrm{d} y
$$

Since $\mathcal{L}^{N}\left(Q \backslash E_{M, k}\right) \rightarrow 0$ as $M \rightarrow+\infty$ (uniformly with respect to $k$ ), the equi-integrability of $\left\{\left|\nabla \bar{w}_{k}\right|^{p}\right\}$ and the $p$-growth condition (2.2) imply

$$
\sup _{k \in \mathbb{N}} \int_{Q \backslash E_{M, k}} \bar{f}\left(\frac{y}{\delta_{k}}, s_{0}, \nabla \bar{w}_{k}\right) \mathrm{d} y \leq \beta^{\prime} \sup _{k \in \mathbb{N}} \int_{Q \backslash E_{M, k}}\left(1+\left|\nabla \bar{w}_{k}\right|^{p}\right) \mathrm{d} y \longrightarrow 0 \quad \text { as } M \rightarrow+\infty .
$$

Plugging this estimate in (5.7) leads to

$$
\frac{\mathrm{d} \mu}{\mathrm{d} \mathcal{L}^{N}}\left(x_{0}\right) \geq \limsup _{k \rightarrow+\infty} \int_{Q} \bar{f}\left(\frac{y}{\delta_{k}}, s_{0}, \nabla \bar{w}_{k}\right) \mathrm{d} y .
$$


Since $\bar{w}_{k} \rightarrow v_{0}$ in $L^{p}\left(Q ; \mathbb{R}^{d}\right)$, we can invoke standard homogenization results (see, e.g., [8], Thm. 14.5) to infer that

$$
\limsup _{k \rightarrow+\infty} \int_{Q} \bar{f}\left(\frac{y}{\delta_{k}}, s_{0}, \nabla \bar{w}_{k}\right) \mathrm{d} y \geq \int_{Q} \bar{f}_{\text {hom }}\left(s_{0}, \nabla v_{0}\right) \mathrm{d} y=\bar{f}_{\text {hom }}\left(s_{0}, \xi_{0}\right) .
$$

In view of Proposition 2.1 we finally conclude

$$
\frac{\mathrm{d} \mu}{\mathrm{d} \mathcal{L}^{N}}\left(x_{0}\right) \geq \bar{f}_{\mathrm{hom}}\left(s_{0}, \xi_{0}\right)=T f_{\mathrm{hom}}\left(s_{0}, \xi_{0}\right),
$$

and the proof is complete.

\subsection{The case of linear growth}

We now treat the case $p=1$ assuming that the function $u$ belongs to $W^{1,1}(\Omega ; \mathcal{M})$. In contrast with the case $p>1$, there is no equi-integrability result as the Decomposition Lemma. We follow here the approach of [15].

Lemma 5.2. Assume $p=1$. Then $\mathcal{F}(u) \geq \mathcal{F}_{\text {hom }}(u)$ for every $u \in W^{1,1}(\Omega ; \mathcal{M})$.

Proof. Let $u \in W^{1,1}(\Omega ; \mathcal{M})$. By a standard diagonal argument, we first obtain a subsequence $\left\{\varepsilon_{n}\right\}$ (not relabeled) and $\left\{u_{n}\right\} \subset W^{1,1}(\Omega ; \mathcal{M})$ such that $u_{n} \rightarrow u$ in $L^{1}\left(\Omega ; \mathbb{R}^{d}\right)$ and

$$
\mathcal{F}(u)=\lim _{n \rightarrow+\infty} \int_{\Omega} f\left(\frac{x}{\varepsilon_{n}}, \nabla u_{n}\right) \mathrm{d} x<+\infty .
$$

Up to the extraction of a further subsequence, we may assume that there exists a nonnegative Radon measure $\mu \in \mathcal{M}(\Omega)$ such that

$$
f\left(\frac{\cdot}{\varepsilon_{n}}, \nabla u_{n}\right) \mathcal{L}^{N}\llcorner\Omega \stackrel{*}{\rightarrow} \mu \quad \text { in } \mathcal{M}(\Omega) .
$$

Hence it is enough to prove that $\mu(\Omega) \geq \mathcal{F}_{\text {hom }}(u)$. As in the proof of Lemma 5.1, it suffices to show that

$$
\frac{\mathrm{d} \mu}{\mathrm{d} \mathcal{L}^{N}}\left(x_{0}\right) \geq T f_{\text {hom }}\left(u\left(x_{0}\right), \nabla u\left(x_{0}\right)\right) \quad \text { for } \mathcal{L}^{N} \text {-a.e. } x_{0} \in \Omega .
$$

The proof will be divided into three steps. We first apply the blow-up method which reduces the study to affine limiting functions. Then we reproduce the argument of [15] which enables us to replace the original sequence by a uniformly converging one without increasing the energy. We will conclude using a classical homogenization result.

Step 1. Select a point $x_{0} \in \Omega$ which is a Lebesgue point of $u$ and $\nabla u$, a point of approximate differentiability of $u$ (so that $u\left(x_{0}\right) \in \mathcal{M}, \nabla u\left(x_{0}\right) \in\left[T_{u\left(x_{0}\right)}(\mathcal{M})\right]^{N}$ ) and such that the Radon-Nikodým derivative of $\mu$ with respect to the Lebesgue measure $\mathcal{L}^{N}$ exists and is finite. Note that $\mathcal{L}^{N}$-almost every points $x_{0}$ in $\Omega$ satisfy these properties. We write $s_{0}:=u\left(x_{0}\right)$ and $\xi_{0}:=\nabla u\left(x_{0}\right)$.

Up to a subsequence, we may assume that there exists a nonnegative Radon measure $\lambda \in \mathcal{M}(\Omega)$ such that $\left(1+\left|\nabla u_{n}\right|\right) \mathcal{L}^{N}\left\llcorner\Omega \stackrel{*}{\rightarrow} \lambda\right.$ in $\mathcal{M}(\Omega)$. Consider a sequence $\left\{\rho_{k}\right\} \searrow 0^{+}$such that $Q\left(x_{0}, 2 \rho_{k}\right) \subset \Omega$ and $\mu\left(\partial Q\left(x_{0}, \rho_{k}\right)\right)=$ $\lambda\left(\partial Q\left(x_{0}, \rho_{k}\right)\right)=0$ for each $k \in \mathbb{N}$. Then (5.8) yields

$$
\mu\left(Q\left(x_{0}, \rho_{k}\right)\right)=\lim _{n \rightarrow+\infty} \int_{Q\left(x_{0}, \rho_{k}\right)} f\left(\frac{x}{\varepsilon_{n}}, \nabla u_{n}\right) \mathrm{d} x .
$$


Set $\tau_{n}:=\varepsilon_{n}\left[x_{0} / \varepsilon_{n}\right] \in \varepsilon_{n} \mathbb{Z}^{N}$. Since $\tau_{n} \rightarrow x_{0}$, given $r \in(1,2)$ we have $Q\left(\tau_{n}, \rho_{k}\right) \subset Q\left(x_{0}, r \rho_{k}\right)$ whenever $n$ is large enough, and we may define for $x \in Q\left(0, \rho_{k}\right), v_{n}(x):=u_{n}\left(x+\tau_{n}\right)$. By continuity of the translation in $L^{1}$, we get that

$$
\begin{aligned}
\int_{Q\left(0, \rho_{k}\right)}\left|v_{n}(x)-u\left(x+x_{0}\right)\right| \mathrm{d} x & =\int_{Q\left(\tau_{n}, \rho_{k}\right)}\left|u_{n}(x)-u\left(x+x_{0}-\tau_{n}\right)\right| \mathrm{d} x \\
& \leq \int_{Q\left(x_{0}, r \rho_{k}\right)}\left|u_{n}(x)-u\left(x+x_{0}-\tau_{n}\right)\right| \mathrm{d} x \underset{n \rightarrow+\infty}{\longrightarrow} 0 .
\end{aligned}
$$

Changing variable in (5.9) and using the periodicity condition $\left(H_{1}\right)$ of $f(\cdot, \xi)$ and the growth condition $\left(H_{2}\right)$, we are led to

$$
\begin{aligned}
\mu\left(Q\left(x_{0}, \rho_{k}\right)\right) & =\lim _{n \rightarrow+\infty} \int_{Q\left(x_{0}-\tau_{n}, \rho_{k}\right)} f\left(\frac{x+\tau_{n}}{\varepsilon_{n}}, \nabla u_{n}\left(x+\tau_{n}\right)\right) \mathrm{d} x \\
& =\lim _{n \rightarrow+\infty} \int_{Q\left(x_{0}-\tau_{n}, \rho_{k}\right)} f\left(\frac{x}{\varepsilon_{n}}, \nabla v_{n}\right) \mathrm{d} x \\
& \geq \limsup _{n \rightarrow+\infty} \int_{Q\left(0, \rho_{k}\right)} f\left(\frac{x}{\varepsilon_{n}}, \nabla v_{n}\right) \mathrm{d} x-\beta \limsup _{n \rightarrow+\infty} \int_{Q\left(\tau_{n}, \rho_{k}\right) \backslash Q\left(x_{0}, \rho_{k}\right)}\left(1+\left|\nabla u_{n}\right|\right) \mathrm{d} x .
\end{aligned}
$$

On the other hand, by our choice of $\rho_{k}$,

$$
\begin{aligned}
\limsup _{n \rightarrow+\infty} \int_{Q\left(\tau_{n}, \rho_{k}\right) \backslash Q\left(x_{0}, \rho_{k}\right)}\left(1+\left|\nabla u_{n}\right|\right) \mathrm{d} x & \leq \limsup _{r \rightarrow 1^{+}} \limsup _{n \rightarrow+\infty} \int_{Q\left(x_{0}, r \rho_{k}\right) \backslash Q\left(x_{0}, \rho_{k}\right)}\left(1+\left|\nabla u_{n}\right|\right) \mathrm{d} x \\
& \leq \limsup _{r \rightarrow 1^{+}} \lambda\left(\overline{Q\left(x_{0}, r \rho_{k}\right) \backslash Q\left(x_{0}, \rho_{k}\right)}\right) \\
& \leq \lambda\left(\partial Q\left(x_{0}, \rho_{k}\right)\right)=0
\end{aligned}
$$

so that the last term in (5.11) vanishes. Hence

$$
\mu\left(Q\left(x_{0}, \rho_{k}\right)\right) \geq \limsup _{n \rightarrow+\infty} \int_{Q\left(0, \rho_{k}\right)} f\left(\frac{x}{\varepsilon_{n}}, \nabla v_{n}\right) \mathrm{d} x
$$

where $\left\{v_{n}\right\} \subset W^{1,1}\left(Q\left(0, \rho_{k}\right) ; \mathcal{M}\right)$ satisfies $v_{n} \rightarrow u\left(x_{0}+\cdot\right)$ in $L^{1}\left(Q\left(0, \rho_{k}\right) ; \mathbb{R}^{d}\right)$ by $(5.10)$.

Now we consider for every $n$, a sequence $\left\{v_{n, j}\right\} \subset \mathcal{C}^{\infty}\left(\overline{Q\left(0, \rho_{k}\right)} ; \mathbb{R}^{d}\right)$ such that $v_{n, j} \rightarrow v_{n}$ in $W^{1,1}\left(Q\left(0, \rho_{k}\right) ; \mathbb{R}^{d}\right)$, $v_{n, j} \rightarrow v_{n}$ and $\nabla v_{n, j} \rightarrow \nabla v_{n}$ a.e. in $Q\left(0, \rho_{k}\right)$ as $j \rightarrow+\infty$ (we emphasize that in general, $v_{n, j}$ is not $\mathcal{M}$-valued). Considering the integrand $g$ given by Lemma 2.1, one may check

$$
\lim _{j \rightarrow+\infty} \int_{Q\left(0, \rho_{k}\right)} g\left(\frac{x}{\varepsilon_{n}}, v_{n, j}, \nabla v_{n, j}\right) \mathrm{d} x=\int_{Q\left(0, \rho_{k}\right)} g\left(\frac{x}{\varepsilon_{n}}, v_{n}, \nabla v_{n}\right) \mathrm{d} x=\int_{Q\left(0, \rho_{k}\right)} f\left(\frac{x}{\varepsilon_{n}}, \nabla v_{n}\right) \mathrm{d} x
$$

so that we can find a diagonal sequence $\bar{v}_{n}:=v_{n, j_{n}}$ satisfying $\bar{v}_{n} \rightarrow u\left(x_{0}+\cdot\right)$ in $L^{1}\left(Q\left(0, \rho_{k}\right) ; \mathbb{R}^{d}\right)$ and

$$
\mu\left(Q\left(x_{0}, \rho_{k}\right)\right) \geq \limsup _{n \rightarrow+\infty} \int_{Q\left(0, \rho_{k}\right)} g\left(\frac{x}{\varepsilon_{n}}, \bar{v}_{n}, \nabla \bar{v}_{n}\right) \mathrm{d} x .
$$


Changing variable in (5.12) yields

$$
\begin{aligned}
\frac{\mathrm{d} \mu}{\mathrm{d} \mathcal{L}^{N}}\left(x_{0}\right) & \geq \limsup _{k \rightarrow+\infty} \limsup _{n \rightarrow+\infty} \int_{Q} g\left(\frac{\rho_{k} x}{\varepsilon_{n}}, \bar{v}_{n}\left(\rho_{k} x\right), \nabla \bar{v}_{n}\left(\rho_{k} x\right)\right) \mathrm{d} x \\
& =\limsup _{k \rightarrow+\infty} \limsup _{n \rightarrow+\infty} \int_{Q} g\left(\frac{\rho_{k} x}{\varepsilon_{n}}, s_{0}+\rho_{k} w_{n, k}, \nabla w_{n, k}\right) \mathrm{d} x,
\end{aligned}
$$

where we have set $w_{n, k}(x):=\left[\bar{v}_{n}\left(\rho_{k} x\right)-s_{0}\right] / \rho_{k}$. Since $x_{0}$ is a point of approximate differentiability of $u$ and $\bar{v}_{n} \rightarrow u\left(x_{0}+\cdot\right)$ in $L^{1}\left(Q\left(0, \rho_{k}\right) ; \mathbb{R}^{d}\right)$, we have

$$
\lim _{k \rightarrow+\infty} \lim _{n \rightarrow+\infty} \int_{Q}\left|w_{n, k}(x)-\xi_{0} x\right| \mathrm{d} x=\lim _{k \rightarrow+\infty} \int_{Q\left(x_{0}, \rho_{k}\right)} \frac{\left|u(y)-s_{0}-\xi_{0}\left(y-x_{0}\right)\right|}{\rho_{k}^{N+1}} \mathrm{~d} y=0 .
$$

In view of (5.13) and (5.14), we can find a diagonal sequence $\varepsilon_{n_{k}}<\rho_{k}^{2}$ such that $w_{k}:=w_{n_{k}, k} \rightarrow w_{0}$ in $L^{1}\left(Q ; \mathbb{R}^{d}\right)$ with $w_{0}(x):=\xi_{0} x$, and

$$
\frac{\mathrm{d} \mu}{\mathrm{d} \mathcal{L}^{N}}\left(x_{0}\right) \geq \limsup _{k \rightarrow+\infty} \int_{Q} g\left(\frac{x}{\delta_{k}}, s_{0}+\rho_{k} w_{k}, \nabla w_{k}\right) \mathrm{d} x,
$$

where $\delta_{k}:=\varepsilon_{n_{k}} / \rho_{k} \rightarrow 0$.

Step 2. We now argue as in Step 3 of the proof of [15], Theorem 2.1, to show that there exists a sequence $\left\{\bar{w}_{k}\right\} \subset W^{1, \infty}\left(Q ; \mathbb{R}^{d}\right)$ such that $\bar{w}_{k} \rightarrow w_{0}$ in $L^{\infty}\left(Q ; \mathbb{R}^{d}\right),\left\{\bar{w}_{k}\right\}$ is uniformly bounded in $W^{1,1}\left(Q ; \mathbb{R}^{d}\right)$ and

$$
\frac{\mathrm{d} \mu}{\mathrm{d} \mathcal{L}^{N}}\left(x_{0}\right) \geq \limsup _{k \rightarrow+\infty} \int_{Q} g\left(\frac{x}{\delta_{k}}, s_{0}+\rho_{k} \bar{w}_{k}, \nabla \bar{w}_{k}\right) \mathrm{d} x .
$$

Given $0<s<t$, let $\zeta_{s, t} \in \mathcal{C}_{c}^{\infty}(\mathbb{R} ;[0,1])$ be a cut-off function satisfying $\zeta_{s, t}(\tau)=1$ if $|\tau| \leq s, \zeta_{s, t}(\tau)=0$ if $|\tau| \geq t$ and $\left|\zeta_{s, t}^{\prime}\right| \leq C /(t-s)$. Define

$$
w_{s, t}^{k}:=w_{0}+\zeta_{s, t}\left(\left|w_{k}-w_{0}\right|\right)\left(w_{k}-w_{0}\right)
$$

Obviously,

and the Chain Rule formula gives

$$
\left\|w_{s, t}^{k}-w_{0}\right\|_{L^{\infty}\left(Q ; \mathbb{R}^{d}\right)} \leq t
$$

$$
\nabla w_{s, t}^{k}=\nabla w_{0}+\zeta_{s, t}\left(\left|w_{k}-w_{0}\right|\right)\left(\nabla w_{k}-\nabla w_{0}\right)+\zeta_{s, t}^{\prime}\left(\left|w_{k}-w_{0}\right|\right)\left(w_{k}-w_{0}\right) \otimes \nabla\left|w_{k}-w_{0}\right| .
$$

In particular,

$$
\begin{aligned}
\int_{Q} g\left(\frac{x}{\delta_{k}}, s_{0}+\rho_{k} w_{s, t}^{k}, \nabla w_{s, t}^{k}\right) \mathrm{d} x= & \int_{\left\{\left|w_{k}-w_{0}\right| \leq s\right\}} g\left(\frac{x}{\delta_{k}}, s_{0}+\rho_{k} w_{k}, \nabla w_{k}\right) \mathrm{d} x \\
& +\int_{\left\{s<\left|w_{k}-w_{0}\right| \leq t\right\}} g\left(\frac{x}{\delta_{k}}, s_{0}+\rho_{k} w_{s, t}^{k}, \nabla w_{s, t}^{k}\right) \mathrm{d} x \\
& \quad+\int_{\left\{\left|w_{k}-w_{0}\right|>t\right\}} g\left(\frac{x}{\delta_{k}}, s_{0}+\rho_{k} w_{0}, \xi_{0}\right) \mathrm{d} x
\end{aligned}
$$

From the growth condition (2.7), we infer that

$$
\int_{\left\{\left|w_{k}-w_{0}\right|>t\right\}} g\left(\frac{x}{\delta_{k}}, s_{0}+\rho_{k} w_{0}, \xi_{0}\right) \mathrm{d} x \leq \beta^{\prime}\left(1+\left|\xi_{0}\right|\right) \mathcal{L}^{N}\left(\left\{\left|w_{k}-w_{0}\right|>t\right\}\right),
$$


and (5.18) yields

$$
\begin{aligned}
\int_{\left\{s<\left|w_{k}-w_{0}\right| \leq t\right\}} g\left(\frac{x}{\delta_{k}}, s_{0}+\rho_{k} w_{s, t}^{k}, \nabla w_{s, t}^{k}\right) \mathrm{d} x \leq C \int_{\left\{s<\left|w_{k}-w_{0}\right| \leq t\right\}} & \left(1+\left|\nabla w_{k}-\xi_{0}\right|\right) \mathrm{d} x \\
& +\frac{C}{t-s} \int_{\left\{s<\left|w_{k}-w_{0}\right| \leq t\right\}}\left|w_{k}-w_{0}\right||\nabla| w_{k}-w_{0}|| \mathrm{d} x
\end{aligned}
$$

Observe that for $\mathcal{L}^{1}$-a.e. $t>0$,

$$
\lim _{s \rightarrow t^{-}} \int_{\left\{s<\left|w_{k}-w_{0}\right| \leq t\right\}}\left(1+\left|\nabla w_{k}-\xi_{0}\right|\right) \mathrm{d} y \leq C_{k} \lim _{s \rightarrow t^{-}} \mathcal{L}^{N}\left(\left\{s<\left|w_{k}-w_{0}\right| \leq t\right\}\right)=0,
$$

and by the Coarea formula,

$$
\begin{aligned}
\lim _{s \rightarrow t^{-}} \frac{1}{t-s} \int_{\left\{s<\left|w_{k}-w_{0}\right| \leq t\right\}} \mid w_{k} & -w_{0}|| \nabla\left|w_{k}-w_{0}\right| \mid \mathrm{d} x \\
& =\lim _{s \rightarrow t^{-}} \frac{1}{t-s} \int_{s}^{t} \tau \mathcal{H}^{N-1}\left(\left\{\left|w_{k}-w_{0}\right|=\tau\right\}\right) \mathrm{d} \tau=t \mathcal{H}^{N-1}\left(\left\{\left|w_{k}-w_{0}\right|=t\right\}\right)
\end{aligned}
$$

Moreover, in view of (2.7) and (5.15) we infer that

$$
\int_{Q}|\nabla| w_{k}-w_{0}|| \mathrm{d} x \leq C \int_{Q}\left(1+\left|\nabla w_{k}\right|\right) \mathrm{d} y \leq C_{0}
$$

Applying [15], Lemma 2.6, there exists $t_{k} \in\left(\left\|w_{k}-w_{0}\right\|_{L^{1}\left(Q ; \mathbb{R}^{d}\right)}^{1 / 2},\left\|w_{k}-w_{0}\right\|_{L^{1}\left(Q ; \mathbb{R}^{d}\right)}^{1 / 3}\right)$ such that $(5.22)$ and $(5.23)$ hold with $t=t_{k}$, and

$$
t_{k} \mathcal{H}^{N-1}\left(\left\{\left|w_{k}-w_{0}\right|=t_{k}\right\}\right) \leq \frac{C_{0}}{\ln \left(\left\|w_{k}-w_{0}\right\|_{L^{1}\left(Q ; \mathbb{R}^{d}\right)}^{-1 / 6}\right)} .
$$

According to $(5.22),(5.23)$ and $(5.24)$, there exists $s_{k} \in\left(\left\|w_{k}-w_{0}\right\|_{L^{1}\left(Q ; \mathbb{R}^{d}\right)}^{1 / 2}, t_{k}\right)$ such that

$$
\int_{\left\{s_{k}<\left|w_{k}-w_{0}\right| \leq t_{k}\right\}}\left(1+\left|\nabla w_{k}-\xi_{0}\right|\right) \mathrm{d} x \leq \frac{1}{k}
$$

and

$$
\frac{1}{t_{k}-s_{k}} \int_{\left\{s_{k}<\left|w_{k}-w_{0}\right| \leq t_{k}\right\}}\left|w_{k}-w_{0}\right||\nabla| w_{k}-w_{0}|| \mathrm{d} x \leq \frac{C_{0}}{\ln \left(\left\|w_{k}-w_{0}\right\|_{L^{1}\left(Q ; \mathbb{R}^{d}\right)}^{-1 / 6}\right)}+\frac{1}{k},
$$

while (5.20) together with Chebyshev inequality yields

$$
\int_{\left\{\left|w_{k}-w_{0}\right|>t_{k}\right\}} g\left(\frac{x}{\delta_{k}}, s_{0}+\rho_{k} w_{0}, \xi_{0}\right) \mathrm{d} y \leq C\left\|w_{k}-w_{0}\right\|_{L^{1}\left(Q ; \mathbb{R}^{d}\right)}^{1 / 2} .
$$

Define now $\bar{w}_{k}:=w_{s_{k}, t_{k}}^{k}$ so that $\bar{w}_{k} \rightarrow w_{0}$ in $L^{\infty}\left(Q ; \mathbb{R}^{d}\right)$ by (5.17). Moreover, gathering (5.19), (5.21), (5.25), (5.26) and (5.27), we deduce

$$
\limsup _{k \rightarrow+\infty} \int_{Q} g\left(\frac{x}{\delta_{k}}, s_{0}+\rho_{k} \bar{w}_{k}, \nabla \bar{w}_{k}\right) \mathrm{d} x \leq \limsup _{k \rightarrow+\infty} \int_{Q} g\left(\frac{x}{\delta_{k}}, s_{0}+\rho_{k} w_{k}, \nabla w_{k}\right) \mathrm{d} x,
$$


which proves (5.16). The fact that $\left\{\nabla \bar{w}_{k}\right\}$ is uniformly bounded in $L^{1}\left(Q ; \mathbb{R}^{d \times N}\right)$ is a consequence of (5.16) and the coercivity condition (2.7).

Step 3. Since $\left\{\left\|\bar{w}_{k}\right\|_{L^{\infty}\left(Q ; \mathbb{R}^{d}\right)}\right\}$ and $\left\{\left\|\nabla \bar{w}_{k}\right\|_{L^{1}\left(Q ; \mathbb{R}^{d \times N}\right)}\right\}$ are uniformly bounded, we derive from (2.8) that

$$
\lim _{k \rightarrow+\infty} \int_{Q}\left|g\left(\frac{x}{\delta_{k}}, s_{0}+\rho_{k} \bar{w}_{k}, \nabla \bar{w}_{k}\right)-g\left(\frac{x}{\delta_{k}}, s_{0}, \nabla \bar{w}_{k}\right)\right| \mathrm{d} x=0 .
$$

In view of (5.16), it leads to

$$
\frac{\mathrm{d} \mu}{\mathrm{d} \mathcal{L}^{N}}\left(x_{0}\right) \geq \lim _{k \rightarrow+\infty} \int_{Q} g\left(\frac{x}{\delta_{k}}, s_{0}, \nabla \bar{w}_{k}\right) \mathrm{d} x .
$$

Using standard homogenization results (see e.g., [8], Thm. 14.5) together with (2.10), we finally conclude that

$$
\frac{\mathrm{d} \mu}{\mathrm{d} \mathcal{L}^{N}}\left(x_{0}\right) \geq g_{\mathrm{hom}}\left(s_{0}, \xi_{0}\right)=T f_{\mathrm{hom}}\left(s_{0}, \xi_{0}\right),
$$

which completes the proof of the lemma.

\subsection{Proof of Theorems 1.1 and 1.2 completed}

Since $L^{p}\left(\Omega ; \mathbb{R}^{d}\right)$ is separable $(1 \leq p<+\infty)$, there exists a subsequence $\left\{\varepsilon_{n_{k}}\right\}$ such that $\mathcal{F}$ is the $\Gamma$-limit of $\left\{\mathcal{F}_{\varepsilon_{n_{k}}}\right\}$ for the strong $L^{p}\left(\Omega ; \mathbb{R}^{d}\right)$-topology (see [13], Thm. 8.5).

Case $p>1$. In view of $\left(H_{2}\right)$ and the closure of the pointwise constraint under strong $L^{p}$-convergence, we have $\mathcal{F}(u)<+\infty$ if and only if $u \in W^{1, p}(\Omega ; \mathcal{M})$. Hence, as a consequence of Lemmas 4.1 and 5.1, the functionals $\left\{\mathcal{F}_{\varepsilon_{n_{k}}}\right\} \Gamma$-converge to $\mathcal{F}_{\text {hom }}$ in $L^{p}\left(\Omega ; \mathbb{R}^{d}\right)$. Since the $\Gamma$-limit does not depend on the extracted subsequence, we get in light of [13], Proposition 8.3, that the whole sequence $\left\{\mathcal{F}_{\mathcal{E}_{n}}\right\} \Gamma$-converges to $\mathcal{F}_{\text {hom }}$.

Case $p=1$. As a consequence of Lemmas 4.1 and 5.2, the functionals $\left\{\mathcal{F}_{\varepsilon_{n_{k}}}\right\} \Gamma$-converge to $\mathcal{F}_{\text {hom }}$ in $W^{1,1}(\Omega ; \mathcal{M})$. Again, the $\Gamma$-limit does not depend on the extracted subsequence, so that the whole sequence $\left\{\mathcal{F}_{\varepsilon_{n}}\right\} \Gamma$-converges to $\mathcal{F}_{\text {hom }}$ in $W^{1,1}(\Omega ; \mathcal{M})$.

Acknowledgements. The authors wish to thank Roberto Alicandro, Pierre Bousquet, Giovanni Leoni and Domenico Mucci for several interesting discussions on the subject. This work was initiated while V. Millot was visiting the department of Functional Analysis and Applications at S.I.S.S.A., he thanks G. Dal Maso and the whole department for the warm hospitality. The research of J.-F. Babadjian was partially supported by the Marie Curie Research Training Network MRTN-CT-2004-505226 "Multi-scale modelling and characterisation for phase transformations in advanced materials" (MULTIMAT). V. Millot was partially supported by the Center for Nonlinear Analysis (CNA) under the National Science Foundation Grant No. 0405343.

\section{REFERENCES}

[1] R. Alicandro and C. Leone, 3D-2D asymptotic analysis for micromagnetic energies. ESAIM: COCV 6 (2001) $489-498$.

[2] L. Ambrosio and G. Dal Maso, On the relaxation in $B V\left(\Omega ; \mathbb{R}^{m}\right)$ of quasiconvex integrals. J. Funct. Anal. 109 (1992) $76-97$.

[3] J.-F. Babadjian and V. Millot, Homogenization of variational problems in manifold valued BV-spaces. Calc. Var. Part. Diff. Eq. 36 (2009) $7-47$.

[4] F. Béthuel, The approximation problem for Sobolev maps between two manifolds. Acta Math. 167 (1991) $153-206$.

[5] F. Béthuel and X. Zheng, Density of smooth functions between two manifolds in Sobolev spaces. J. Funct. Anal. 80 (1988) 60-75.

[6] F. Béthuel, H. Brézis and J.M. Coron, Relaxed energies for harmonic maps, in Variational methods, Paris (1988), H. Berestycki, J.M. Coron and I. Ekeland Eds., Progress in Nonlinear Differential Equations and Their Applications 4, Birkhäuser, Boston (1990) 37-52.

[7] A. Braides, Homogenization of some almost periodic coercive functional. Rend. Accad. Naz. Sci. XL 103 (1985) 313-322.

[8] A. Braides and A. Defranceschi, Homogenization of multiple integrals, Oxford Lecture Series in Mathematics and its Applications 12. Oxford University Press, New York (1998). 
[9] A. Braides, A. Defranceschi and E. Vitali, Homogenization of free discontinuity problems. Arch. Rational Mech. Anal. 135 (1996) $297-356$.

[10] H. Brézis, J.M. Coron and E.H. Lieb, Harmonic maps with defects. Comm. Math. Phys. 107 (1986) 649-705.

[11] B. Dacorogna, Direct methods in the calculus of variations. Springer-Verlag (1989).

[12] B. Dacorogna, I. Fonseca, J. Malý and K. Trivisa, Manifold constrained variational problems. Calc. Var. Part. Diff. Eq. 9 (1999) 185-206.

[13] G. Dal Maso, An Introdution to $\Gamma$-convergence. Birkhäuser, Boston (1993).

[14] I. Ekeland and R. Temam, Analyse convexe et problèmes variationnels. Dunod, Gauthiers-Villars, Paris (1974).

[15] I. Fonseca and S. Müller, Quasiconvex integrands and lower semicontinuity in L ${ }^{1}$. SIAM J. Math. Anal. 23 (1992) $1081-1098$.

[16] I. Fonseca and S. Müller, Relaxation of quasiconvex functionals in $B V\left(\Omega ; \mathbb{R}^{p}\right)$ for integrands $f(x, u, \nabla u)$. Arch. Rational Mech. Anal. 123 (1993) 1-49.

[17] I. Fonseca, S. Müller and P. Pedregal, Analysis of concentration and oscillation effects generated by gradients. SIAM J. Math. Anal. 29 (1998) 736-756.

[18] M. Giaquinta, L. Modica and J. Souček, Cartesian currents in the calculus of variations, Modern surveys in Mathematics 37-38. Springer-Verlag, Berlin (1998).

[19] M. Giaquinta, L. Modica and D. Mucci, The relaxed Dirichlet energy of manifold constrained mappings. Adv. Calc. Var. 1 (2008) $1-51$.

[20] P. Marcellini, Periodic solutions and homogenization of nonlinear variational problems. Ann. Mat. Pura Appl. (4) 117 (1978) $139-152$.

[21] S. Müller, Homogenization of nonconvex integral functionals and cellular elastic materials. Arch. Rational Mech. Anal. 99 (1987) 189-212. 